\title{
Independência e o modelo de preferências Bewley Variacional
}

\author{
Flávia Sousa Teles da Cunha
}

\author{
DISSERTAÇÃO APRESENTADA \\ Instituto DE MATEMÁtica E EstatísticA \\ UNIVERSIDADE DE SÃO PAULO \\ PARA \\ OBTENÇẪO DO TÍTULO \\ MESTRE EM CIÊNCIAS
}

Programa: Matemática Aplicada

Orientador: Prof. Dr. Fábio Armando Tal

Durante o desenvolvimento deste trabalho o autor recebeu auxílio financeiro do $\mathrm{CNPq}$

São Paulo, junho de 2016 


\section{Independência e o modelo de preferências Bewley Variacional}

Esta versão da dissertação contém as correções e alterações sugeridas pela Comissão Julgadora durante a defesa da versão original do trabalho, realizada em 11/05/2016. Uma cópia da versão original está disponível no

Instituto de Matemática e Estatística da Universidade de São Paulo.

Comissão Julgadora:

- Prof. Dr. Fábio Armando Tal (orientador) - IME-USP

- Prof. Dr. José Heleno Faro - IMPA

- Prof. Dr. Antônio Bruno de Carvalho Morales - FEA-USP 


\section{Capítulo 1}

\section{Agradecimentos}

Agradeço a minha falecida mãe Maria Luiza por ter me ensinado o valor do conhecimento e o apreço pela Matemática. 


\section{Capítulo 2}

\section{Resumo}

CUNHA, Flávia S. T. Independência e o modelo de preferências Bewley Variacional. 2016. 47 f. Dissertação (Mestrado) - Instituto de Matemática e Estatística, Universidade de São Paulo, São Paulo, 2016.

Modelos de escolha perante incertezas incorporando ambiguidade se tornaram uma tema importante de pesquisa após a apresentação do Paradoxo de Ellsberg. O intuito do presente trabalho é contribuir para essa área do conhecimento através de uma análise mais detalhada do modelo de preferências Bewley Variacional proposto por Faro [8]. Primeiro foi feita uma revisão bibliográfica da literatura, culminando no modelo em questão e finalmente sugerindo um aproveitamento no entendimento do axioma da independência.

Palavras-chave: ambiguidade, Paradoxo de Ellsberg, incertezas, independência . 


\section{Capítulo 3}

\section{Abstract}

CUNHA, Flávia S. T. Independence and Variational Bewley Preferences. 2016. 47 f. Dissertação (Mestrado) - Instituto de Matemática e Estatística, Universidade de São Paulo, São Paulo, 2016.

Models of choice with uncertainty that allows for ambiguity to be present have become an important subject of research after Ellsberg's Paradox. This work aims to contribute to this area of knowledge through a more detailed analysis of the model Variational Bewley Preferences proposed by Faro [8]. First we present a survey of the literature, then analyse and finally suggest a further understanding of Faro's model with respect to the independence axiom.

Keywords: ambiguity, Ellsbergs Paradox, uncertainty, independence. 


\section{Sumário}

1 Agradecimentos

2 Resumo iii

3 Abstract $\quad$ v

4 Introdução 3

5 Revisão Bibliográfica $\quad 5$

5.1 Probabilidade Subjetiva . . . . . . . . . . . . 5

5.2 O Escopo Anscombe-Aumann no Modelo da Utilidade Esperada com Probabilidades Subjetivas . . . . . . . . . 10

5.3 O Paradoxo de Ellsberg . . . . . . . . . . . . . . 12

5.4 O Modelo Maxmin de Utilidade Esperada . . . . . . . . . . 15

5.5 O Modelo de Preferências Variacionais . . . . . . . . . . . . 16

5.6 O Modelo Bewley de Preferências Incompletas . . . . . . . . 18

6 Preferências Bewley Variacionais $\quad 19$

6.1 Escopo do Modelo . . . . . . . . . . . . . . . . . . . 20

6.2 Axiomas ........................ 21

6.3 Representação do Modelo Principal . . . . . . . . . . . . . . . 22

6.4 Extensões a partir de PBV . . . . . . . . . . . . . 23

6.4.1 Preferências Bewley Incompletas . . . . . . . . . . . . 23

6.4.2 Preferências Bewley Variacionais Completas . . . . . . 23

6.4.3 Conexão com Preferências Variacionais . . . . . . . . . 24

7 Independência e Preferências Bewley Variacionais $\quad 25$

8 Conclusão $\quad 29$ 
$\begin{array}{lr}\text { A Apêndice } & 31\end{array}$

A.1 Demonstrações PBV . . . . . . . . . . . . . . . . . 31

A.1.1 Relações Binárias Fundamentais . . . . . . . . . . 31

A.1.2 Lemas Preliminares . . . . . . . . . . . . 31

A.1.3 Resultado Principal . . . . . . . . . . . . 34 


\section{Capítulo 4}

\section{Introdução}

Desde o século passado o estudo das tomadas de decisão têm sido tema recorrente de análise na literatura, conferindo à pesquisa econômica um caráter científico muito mais rigoroso desde então. Muitas contribuições ilustres já foram feitas ao longo dos anos na área; indo desde a formulação axiomática da escolha individual até as contestações da mesma.

A pretensão de modelar o processo individual de decisão perante incertezas não seria possível sem algumas suposições (axiomas) a respeito das características pessoais dos tomadores de decisão (daqui em diante abreviados por TD). A principal delas seria a de racionalidade; ou seja, supõe-se que indivíduos racionais se comportam de maneira lógica o suficiente; sendo possível captar seu processo de escolha através de um modelo matemático. A justificativa de racionalidade nos modelos da literatura é normativa; indivíduos que se enquadram nos axiomas são classificados como racionais e, caso contrário, não seriam porque não se enquadram nos axiomas. Afinal, seria possível comprovar alguma contradição em seu processo de escolha, absurdo que pessoas racionais, por hipótese, não estariam dispostas a cometer.

No entanto, Ellsberg [6] propôs um experimento que contesta tal abordagem. O autor defende que, em situações especiais, TD racionais escolhem deliberadamente agir em contradição com os axiomas; minando a sua natureza de ser. Tal experimento ficou conhecido como Paradoxo de Ellsberg e introduziu um novo fenômeno no estudo da teoria de escolha, o da ambiguidade. Desde então a literatura apresentou inúmeros modelos matemáticos com o objetivo de incorporar a ambiguidade, alguns deles em breve discutidos na revisão bibliográfica.

O foco do presente estudo se encontra em um desses modelos, proposto por Faro [8] e intitulado Preferências Bewley Variacionais (daqui em diante abreviado por PBV). Nele o autor propõe uma nova formulação do funcional de escolha que consegue incluir os principais modelos propostos anteriormente. Assim sendo, o modelo PBV se apresenta como importante generalização dos modelos de escolha sob ambiguidade.

No entanto, o trabalho do autor deixa uma questão em aberto com relação a um importante axioma da teoria da escolha; o da independência. Isso porque ele adota uma versão mais fraca da independência clássica mas, com suposições adicionais, é possível obtê-la novamente e chegar em outros funcionais de escolha de modelos de ambiguidade. Assim, fica o questionamento sobre o grau de enfraquecimento que Faro [8] imputou sobre esse axioma.

Vamos propor duas definições novas e mostrar que juntas, elas resultam na formulação clássica da independência. Depois, veremos que o modelo de 
Preferências Bewley Variacionais atende somente a uma delas; evidenciando assim, o quanto da independência clássica foi perdida.

Este trabalho se encontra organizado da seguinte maneira: começamos com uma retrospectiva da teoria pré Paradoxo de Ellsberg, evoluindo para os modelos de ambiguidade e culminando no PBV; onde serão propostas novas definições, especificamente no que diz respeito a independência. 


\section{Capítulo 5}

\section{Revisão Bibliográfica}

\subsection{Probabilidade Subjetiva}

Na discussão sobre teoria da decisão perante incertezas a seguinte proposição, associada primeiramente a Daniel Bernoulli, resume a hipotética econômica que cerca o processo de escolha. Ela ficou conhecida como Proposição de Bernoulli.

For a reasonable decision-maker, there exists a set of numbers ("vN-M utilities") corresponding to the uncertain outcomes of any given risky proposition or "gamble", and a set of numbers (numerical probabilities) corresponding to the events determining these outcomes, such that the ranking of mathematical expectations of utility computed for various gambles on the basis of these numbers will reflect his actual, deliberate preferences among these gambles. [6]

Formalizando o argumento temos que:

Consider any context where a "reasonable decision-maker" faces a choice between a set $A=a_{1}, \ldots, a_{n}$ of options. (I shall assume the set of options is finite). I shall suppose (as Ellsberg does) that from the decision maker's point of view, the outcome $o_{i j}$ of implementing a given option that is of interest to the decision-maker is uncertain. The outcome $o_{i j}$ that will occur is determined by: (1) by the true state $h_{j}$ of the world, and (2) by the option $a_{i}$ chosen.

\begin{tabular}{|c|c|c|c|c|}
\hline Atos/Estados & $h_{1}$ & $h_{2} \ldots$ & $h_{j} \ldots$ & $h_{m}$ \\
\hline \hline$a_{1}$ & $o_{11}$ & $o_{12}$ & $o_{1 j}$ & $o_{1 m}$ \\
\hline$a_{2}$ & $o_{21}$ & $o_{22}$ & $o_{2 j}$ & $o_{2 m}$ \\
\hline$a_{i}$ & $o_{i 1}$ & $o_{i 2}$ & $o_{i j}$ & $o_{i m}$ \\
\hline$a_{n}$ & $o_{n 1}$ & $o_{n 2}$ & $o_{n j}$ & $o_{n m}$ \\
\hline
\end{tabular}

Tabela 5.1: Representação Matricial de um Problema de Decisão

\footnotetext{
${ }^{1}$ Utiliza-se a abreviação vN-M para referência aos autores John von Neumann e Oskar Morgenstern; que no trabalho "Theory of Games and Economic Behaviour" [18] introduziram o tratamento axiomático na teoria da escolha e formalizaram a definição de utilidade como um número real. Utilidades vN-M se referem a esse arcabouço teórico.
} 
According to the Bernoulli Proposition a "reasonable decision maker" should assign real numbers to the outcomes $o_{i j}$ determined by a vN-M utility function $u\left(o_{i j}\right)=u_{i j}$. Such a decisionmaker should assign other numbers to the states according to a real valued probability function $P\left(h_{j} / a_{i}\right)$ defined over the $h_{j}^{\prime} s$ conditional on the $a_{i}$ chosen so that for each $a_{i}$ and $h_{j}$, the following conditions hold:

- $P\left(h_{j} / a_{i}\right)=P\left(h_{j}\right)^{2}$.

- $P\left(h_{j}\right) \geq 0^{3}$.

- $\sum_{j} P\left(h_{j}\right)=1^{4}$. Since $a_{i}$ and $h_{j}$ determine $o_{i j}$

- $P\left(o_{i j} / a_{i}\right)=P\left(h_{j}\right)^{5}$.

The function $u$ is a $v N-M$ utility function in the sense that if there is a suitable probability $P$ defined over the states, the value of each of the $a_{i}^{\prime} s$ is equal to the expected utility $E\left(a_{i}\right)=$ $\sum_{j} P\left(h_{j}\right) u\left(o_{i j}\right) \cdot[7]$.

Foi através do Teorema de Bayes que a comprovação matemática da Proposição de Bernoulli se realizou; o que permitiu sua invocação na modelagem das escolhas. Tal metodologia, na teoria econômica, ficou conhecida como Abordagem Bayesiana.

No entanto, muitos autores duvidavam da existência de uma medida de probabilidade adequada no processo de tomada de decisão quando os eventos analisados não tem probabilidade objetiva. Foi este questionamento que levou Frank Knight [15] a distinguir entre os termos Risco e Incerteza. $\mathrm{O}$ anterior se refere às situações nas quais o indivíduo é defrontado com probabilidades objetivas, que podem ser deduzidas ou determinadas empiricamente; já o posterior se refere às situações em que não há nenhuma base para a medição dessas probabilidades.

Apesar de a Proposição de Bernoulli funcionar com probabilidades objetivas, as decisões cotidianas raramente são acompanhadas de medidas de probabilidade dessa natureza. Assim, cético com relação a sua existência, Savage [17] propôs uma série de postulados para preferências racionais sobre potenciais alternativas que, quando satisfeitos, nos permitem concluir que o TD se comporta como se endossasse uma medida de probabilidade (mesmo quando esta não é empiricamente verificável). Desse modo, o agente segue maximizando a sua utilidade esperada através da utilização dessa distribuição subjetiva de probabilidade. O modelo formalizado em "The Foundation of Statistics" ficou conhecido como Subjective Expected Utility Model, ou Modelo de Utilidade Esperada Subjetiva, (daqui em diante abreviado como UES).

A teoria idealizada por Savage [17], se baseia no comportamento de um indivíduo dito racional com respeito as suas decisões e nessa empreitada se faz necessário que:

When certain maxims are presented for your consideration, you must ask yourself whether you try to behave in accordance with

\footnotetext{
${ }^{2}$ a probabilidade de um estado da natureza independe do ato.

${ }^{3}$ a probabilidade de estados da natureza é sempre não negativa.

4 a soma de todos os possíveis estados da natureza é 1 .

${ }^{5}$ a probabilidade de um outcome $o_{i j}$ dado o ato $a_{i}$, é a probabilidade do estado da natureza $j$ se realizar.
} 
then, or, to put it differently, how you would react if you noticed yourself violating them. ${ }^{6}$ Savage [17].

Na formulação dessa teoria, Savage define a nomenclatura apresentada na Tabela (5.2) a seguir:

\begin{tabular}{|c||l|}
\hline Nomenclatura & Definição \\
\hline \hline o mundo & $\begin{array}{l}\text { objeto sob o qual a pessoa está } \\
\text { fazendo considerações. }\end{array}$ \\
\hline um estado (do mundo) & $\begin{array}{l}\text { uma descrição do mundo, de- } \\
\text { talhando qualquer aspecto re- } \\
\text { levante. }\end{array}$ \\
\hline o verdadeiro (estado do mundo) & $\begin{array}{l}\text { o estado que de fato acontece, } \\
\text { i.e., a descrição verdadeira do } \\
\text { mundo. }\end{array}$ \\
\hline
\end{tabular}

Tabela 5.2: Definições Gerais

Diz-se evento a um conjunto de estados, sendo $S$ o conjunto universal que possui todos os possíveis estados do mundo como elemento. Diremos que tomar uma decisão equivale a decidir entre dois ou mais atos disponíveis. Ao escolher um ato, deve-se levar em consideração as possíveis consequências dependentes de cada estado do mundo; sendo uma consequência qualquer coisa que pode acontecer ao indivíduo. Outras informações importantes se encontram resumidas na Tabela (5.4).

Savage segue com um exemplo bem ilustrativo: Suponha que sua(eu) esposa(o) acabou de quebrar cinco ovos dentro de uma tigela quando você se oferece para terminar o omelete. Um sexto ovo inteiro ainda resta ao lado da tigela. Você deve então escolher uma dentre três ações possíveis: quebrar o sexto ovo na mesma tigela dos restantes, quebrá-lo num recipiente separado ou simplesmeste jogá-lo fora. Dependendo do estado do ovo, cada ato terá uma consequência diferente conforme indicado na tabela abaixo.

\begin{tabular}{|l||l|l|}
\hline \multicolumn{1}{|l||}{} & \multicolumn{2}{c|}{ Estado } \\
\hline Ação & Bom $\left(s_{1}\right)$ & Ruim $\left(s_{2}\right)$ \\
\hline $\begin{array}{l}\text { quebrar na mesma ti- } \\
\text { gela }(f)\end{array}$ & omelete de 6 ovos $f\left(s_{1}\right)$ & $\begin{array}{l}\text { sem omelete e } 5 \text { ovos bons per- } \\
\text { didos } f\left(s_{2}\right)\end{array}$ \\
\hline $\begin{array}{l}\text { quebrar em outro recipi- } \\
\text { ente }(g)\end{array}$ & $\begin{array}{l}\text { omelete de } 6 \text { ovos e outro re- } \\
\text { cipiente para lavar } g\left(s_{1}\right)\end{array}$ & $\begin{array}{l}\text { omelete de } 5 \text { ovos e outro re- } \\
\text { cipiente para lavar } g\left(s_{2}\right)\end{array}$ \\
\hline jogar fora $(h)$ & $\begin{array}{l}\text { omelete de } 5 \text { ovos e 1 ovo bom } \\
\text { jogado fora } h\left(s_{1}\right)\end{array}$ & \begin{tabular}{l} 
omelete de 5 ovos $h\left(s_{2}\right)$ \\
\hline
\end{tabular} \\
\hline
\end{tabular}

Tabela 5.3: Exemplo ilustrando ações, estados e consequências

O conjunto dos atos de um problema será denotado por $\mathcal{F}$; e os atos individuais por $f, g, h$ etc.

Savage então define a nomenclatura apresentada na Tabela (5.4).

Note que se dois atos individuais $f$ e $g$ têm a mesma consequência em todo e qualquer estado, então não há necessidade de diferenciá-los. Formalmente então, um ato é uma função relacionando uma consequência a cada

\footnotetext{
${ }^{6}$ Esse questionamento será essencial para validar o Paradoxo de Ellsberg, o qual demonstra que, mesmo violando um dos postulados de Savage, indivíduos racionais preferem manter a decisão violatória ao invés de mudá-la para uma escolha racional segundo os axiomas.
} 


\begin{tabular}{|c|c|}
\hline Termo & Definição \\
\hline \multicolumn{2}{|l|}{ (Termos básicos) } \\
\hline $\begin{array}{l}\text { Conjunto } \\
A, B, C, \ldots \\
s, s^{\prime}, s^{\prime \prime}, \ldots \\
S \\
0\end{array}$ & $\begin{array}{l}\text { Evento } \\
\text { símbolos genéricos de eventos } \\
\text { símbolos genéricos de estados } \\
\text { o evento universal } \\
\text { o evento vazio }\end{array}$ \\
\hline \multicolumn{2}{|l|}{ (Relações) } \\
\hline $\begin{array}{l}s \in A \\
A \subset B(\text { or } B \supset A) \\
A=B\end{array}$ & $\begin{array}{l}s \text { é um elemento de } A \text {, ie, um estado } \\
\text { em } A \\
A \text { está contido em } B \text {, ie, todo ele- } \\
\text { mento de } A \text { é um elemento de } B \\
A \text { é igual a } B \text {,ie, } A \text { é o mesmo que } \\
B \text {, ie, } A \text { e } B \text { têm exatamente os mes- } \\
\text { mos elementos }\end{array}$ \\
\hline \multicolumn{2}{|l|}{ (Construçôes) } \\
\hline $\begin{array}{l}\text { o complemento de } A \text { com respeito a } \\
S \\
\sim A\end{array}$ & $\begin{array}{l}\text { os elementos de } S \text { que não estão em } \\
A \\
\text { o complemento de } A \text { com respeito a } \\
S\end{array}$ \\
\hline a união dos $A_{i}^{\prime} s$ & $\begin{array}{l}\text { aqueles elementos de } S \text { que são ele- } \\
\text { mentos de pelo menos um conjunto } \\
A_{1}, A_{2}, \ldots\end{array}$ \\
\hline $\begin{array}{l}\cup_{i} A_{i} \\
A \cup B\end{array}$ & $\begin{array}{l}\text { a união dos } A_{i}^{\prime} s \\
\text { a união de } A \text { e } B \text {, ie, aqueles ele- } \\
\text { mentos de } S \text { que são elementos de } \\
A \text { ou } B \text { (possivelmente ambos) }\end{array}$ \\
\hline a inteseção $\operatorname{dos} A_{i}^{\prime} s$ & $\begin{array}{l}\text { aqueles elementos de } S \text { que são ele- } \\
\text { mentos de cada um dos conjuntos } \\
A_{1}, A_{2}, \ldots \\
\text { a interseção dos } A_{i}^{\prime} s \\
\text { a interseção de } A \text { e } B \text {, ie, aqueles } \\
\text { elementos de } S \text { que são elementos } \\
\text { de ambos } A \text { e } B\end{array}$ \\
\hline
\end{tabular}

Tabela 5.4: Nomenclatura Matemática

estado do mundo. Assim $f$ denotará a função que devolve a consequência $f(s)$, correspondente a realização do estado $s$.

O próximo passo do autor será estabelecer uma série de axiomas que buscam definir preferências (de indivíduos racionais) sobre consequências através das preferências sobre os atos.

- P1: A relação $\leq^{7}$ é um ordenamento simples entre atos. Ou seja, ou $x \leq y$ ou $y \leq x$; e se $x \leq y$ e $y \leq z$, então $x \leq z$.

Um desdobramento importante deste postulado é a proposição abaixo.

Proposição 5.1.1. Se $\mathcal{F}$ é um conjunto finito de atos, existe então $f$ e $h$ em $\mathcal{F}$ tais que, para todo $g \in \mathcal{F}, f \leq g \leq h$.

\footnotetext{
${ }^{7} \mathrm{O}$ autor denota por $\leq$ a relação não preferido $a$.
} 
Note que quando $\mathcal{F}$ é finito, pela Proposição (5.1.1) é garantida a existência de um ato $h$ em $F$ de modo que nenhum outro ato em $\mathcal{F}$ é preferível a $h$. Essa proposição pode ser interpretada como um critério lógico consistente em situações de decisão.

- P2: Princípio da Coisa Segura: Se $f, g$ e $f^{\prime}, g^{\prime}$ são tais que

(i) Em $\sim B, f$ tem o mesmo retorno do que $g$ e $f^{\prime}$ tem o mesmo retorno de $g^{\prime}$.

(ii) Em $B, f$ tem o mesmo retorno do que $f^{\prime}$ e $g$ tem o mesmo retorno de $g^{\prime}$.

(iii) $f \leq g$.

Então $f^{\prime} \leq g^{\prime}$.

O axioma acima simplesmente atesta que, ao realizar uma escolha entre dois atos, o TD não leva em consideração aqueles estados da natureza que retornam a mesma consequência para ambos. Veja o exemplo abaixo:

\section{Exemplo 5.1.1.}

\begin{tabular}{|c||c|c|c|}
\hline & $A$ & $B$ & $\bar{A} \cap \bar{B}$ \\
\hline \hline$f$ & $\mathrm{a}$ & $\mathrm{b}$ & $\mathrm{c}$ \\
\hline$g$ & $\mathrm{~b}$ & $\mathrm{a}$ & $\mathrm{c}$ \\
\hline
\end{tabular}

Considere um indivíduo perante o problema de decidir entre as ações $f$ e $g$, com as consequências $a, b$ e $c$ dependentes dos eventos $A$ e $B$ conforme a tabela acima. Caso ele soubesse que a terceira coluna não acontecerá, ele diz que não prefere $g$ a $f$; no entanto, caso soubesse que a terceira coluna irá acontecer, ele continua não preferindo $g$ a $f$ já que nessa coluna os pay-offs são iguais. Assim, como o TD não prefere $g$ a $f$ em qualquer um dos eventos, ele não deve preferir $g$ mesmo sem saber se a terceira coluna irá ou não se realizar.

Savage introduz a seguinte definição:

Definição 5.1.2. Uma relação binária $\leq$ sobre uma família de eventos ${ }^{8}$ é uma probabilidade qualitativa; se e somente se, para quaisquer eventos $B, C, D$

1. $\leq$ é uma ordem fraca.

2. $B \leq C$, se e somente se $B \cup D \leq C \cup D$, dado $B \cap D=C \cap D=0$.

3. $0 \leq B, 0<S$.

Que é complementada pela:

Definição 5.1.3. Uma medida de probabilidade sobre um conjunto $S$ é uma função $p: \Sigma \rightarrow[0,1]^{9}$ atribuindo um número real $P(B)$ a cada $B \subset S$ de forma que:

1. $P(B) \geq 0$ para cada $B$.

2. Se $B \cap C=0, P(B \cup C)=P(B)+P(C)$.

3. $P(S)=1$.

\footnotetext{
${ }^{8} \mathrm{~A}$ notação $A \leq \leq^{\cdot} B$ diz que o evento $A$ não é mais provável do que $B$.

${ }^{9}$ Para $\Sigma=2^{S}$.
} 
De maneira simplificada, Savage irá mostrar que 5.1.2 $\Leftrightarrow$ 5.1.3.

O autor segue com os axiomas:

- P3: Sejam $f \equiv g, f^{\prime} \equiv g^{\prime}$ e $B$ não nulo. Dado $B$, tem-se $f \leq f^{\prime}$ se e somente se $g \leq g^{\prime}$.

- P4: Sejam $f, f^{\prime}, g, g^{\prime}, A, B, f_{A}, f_{B}, g_{A}, g_{B}$ tais que

1. $f^{\prime}<f, g^{\prime}<g$;

2. $\left\{\begin{array}{l}f_{A}(s)=f, g_{A}(s)=g, \quad \text { para } s \in A \\ f_{A}(s)=f^{\prime}, g_{A}(s)=g^{\prime}, \quad \text { para } s \in \sim A ;\end{array}\right.$

3. $\left\{\begin{array}{l}f_{B}(s)=f, g_{B}(s)=g \quad \text { para } s \in B \\ f_{B}(s)=f^{\prime}, g_{B}(s)=g^{\prime} \quad \text { para } s \in \sim B ;\end{array}\right.$

4. $f_{A} \leq f_{B}$ então $g_{A} \leq g_{B}$.

- P5: Existe pelo menos um par de consequências $f, f^{\prime}$ tais que $f^{\prime}<f$.

- P6: Sejam $B, C$ eventos em $S$ tais que $B<\cdot C$, então existe uma partição de $S$ na qual a união de cada elemento com $B$ é menos provável do que $C$.

Em resumo, a partir dos axiomas e definições acima; Savage demonstra que, mesmo na falta de probabilidades objetivas; o agente racional se comporta como se tivesse uma medida de probabilidade subjetiva em seu processo de decisão.

\subsection{O Escopo Anscombe-Aumann no Modelo da Utilidade Esperada com Probabilidades Sub- jetivas}

Em 1963, Anscombe-Aumann [14] (denotados por AA) desenvolveram uma versão do modelo UES que passou a ser comumente utilizada já que seu tratamento matemático é simplificado. A característica básica no escopo AA são atos considerados como funções mapeando estados da natureza em loterias simples ${ }^{10}$; assim, as consequências de ações envolvem probabilidades exógenas. Vale mencionar que este será o escopo adotado pelos modelos subsequentes de ambiguidade, inclusive PBV.

Considera-se o conjunto de probabilidades simples ${ }^{11} X$, sobre um espaço de alternativas $Z$ como o conjunto das consequências no escopo AA. ${ }^{12}$ Existe um espaço dos estados da natureza $S$ dotado de uma algebra de eventos $\Sigma$. Aqui os objetos de escolha são atos do tipo $f: S \rightarrow X$, ou seja, eles assumem valores finitos e são $\Sigma$-mensuráveis. Denota-se por $\mathcal{F}$ o conjunto dos atos desse tipo.

O aspecto chave de $X$ é a convexidade que possibilita a mistura de ações. Dessa forma, dado qualquer $\alpha \in[0,1]$, fica bem definido o ato $(\alpha f+$

\footnotetext{
${ }^{10}$ Uma loteria (simples) é definida por uma distribuição de probabilidade, com suporte finito, sobre o conjunto de consequências $Z$; formalmente: $L=$ $\left\{p: Z \rightarrow[0,1] ; \#\{z \in Z: p(z) \neq 0\}<\infty, \sum_{z \in Z} p(z)=1\right\}$

${ }^{11}$ Considera-se uma probabilidade simples aquela que tem suporte finito.

${ }^{12}$ Desse modo o espaço de consequências é da forma $X=\Delta(Z)$, sendo $Z$ o espaço das alternativas ou dos resultados determinísticos. De modo geral, é possível considerar $X$ como um subconjunto convexo de um espaço vetorial; no entanto, em [14] os autores utilizam o caso particular mencionado anteriormente.
} 
$(1-\alpha) g)(s)=\alpha f(s)+(1-\alpha) g(s)$. Assim, após a realização do estado da natureza $s$, o indivíduo obtém uma realização (com risco mas não incerteza) governada pela loteria $\alpha f(s)+(1-\alpha) g(s)$.

Assim,

The possibility of mixing acts is a key dividend of the assumption that $X$ is the consequence space, which gives the AA setting a vector structure that the Savage setting did not have. The derivation of the subjective expected utility representation in the $A A$ setting is based on this vector structure. [11].

Seja $\succsim$ uma relação binária. Seguem os axiomas:

AA.1 Ordem Fraca: $\succsim$ em $\mathcal{F}$ é completa e transitiva ${ }^{13}$.

AA.2 Monotonicidade: Para qualquer $f, g \in \mathcal{F}$, se $f(s) \succsim_{X} g(s)$ para cada $s \in S$, então $f \succsim g$. Sendo $\succsim_{X}$ a relação binária de preferências restrita a $X$, que consideramos ser induzida pela preferência entre atos constantes.

AA.3 Independência ${ }^{14}$ : Para quaisquer atos $f, g, h \in \mathcal{F}$ e qualquer $0<\alpha<1$, temos

$$
f \succsim g \Leftrightarrow \alpha f+(1-\alpha) h \succsim \alpha g+(1-\alpha) h
$$

De acordo com AA.3, a mistura com um ato comum $h$ não afeta a preferência de um indivíduo sobre dois atos $f, g l$.

AA.4 Continuidade Arquimediana: Sejam $f, g$ e $h$ três atos quaisquer em $\mathcal{F}$ tais que $f \succ g \succ h$. Então, existem $\alpha, \beta \in(0,1)$ tais que $\alpha f+(1-\alpha) h \succ g \succ \beta f+(1-\beta) h$

AA.5 Não Degeneração: Existem $f, g \in \mathcal{F}$ tais que $f \succ g$.

Segue o Teorema da Utilidade Esperada com Probabilidades Subjetivas no escopo AA:

Teorema 5.2.1. ${ }^{15}$ Seja $\succsim$ uma preferência definida em $\mathcal{F}$. As seguintes condições são equivalentes:

(i) $\succsim$ satisfaz AA.1-AA.5.

(ii) Existe uma função não constante $u: X \rightarrow \mathbb{R}$ e uma medida de probabilidade $p: \Sigma \rightarrow[0,1]$ tal que, para todo $f, g \in \mathcal{F}, f \succsim g$ se e somente se

$$
\int_{S}\left(\sum_{x \in \operatorname{supp} f(s)} u(x) f(s)\right) d p(s) \geq \int_{S}\left(\sum_{x \in \operatorname{suppg}(s)} u(x) g(s)\right) d p(s)
$$

Mais adiante, para aliviar notação, será feita referência a $\sum_{x \in \operatorname{supp} f(s)} u(x) f(s)$ apenas como $u(f)$.

Dentro deste arcabouço teórico se desenvolveu a teoria subsequente mais notável sobre tomada de decisão frente a incertezas.

Vale salientar que o modelo proposto por AA [14] forneceu uma abordagem prática à teoria de Savage, além de englobar a teoria da utilidade

\footnotetext{
${ }^{13}$ Dizemos que uma preferência é completa quando o indivíduo é sempre capaz de comparar quaisquer dois atos de $\mathcal{F}$. Já a transitividade afirma que: dados $f, g, h \in \mathcal{F}$; se $f \succsim g, g \succsim h$ então $f \succsim h$

${ }^{14} \mathrm{O}$ axioma da independência no escopo AA tem forma particular já que se baseia na estrutura vetorial do modelo.

${ }^{15}$ Referência em [11] e [14]
} 
desenvolvida por John von Neumann e Oskar Morgenstern [18] quando consideramos atos constantes.

Mais especificamente, um ato $f \in \mathcal{F}$ é constante se, para todo estado da natureza $s \in S$, ele retorna a mesma consequência $x \in X$. Nesses casos é possível identificar o ato $f$ com a sua consequência $x$, que no escopo AA é uma loteria simples.

Ao considerar atos constantes, as únicas probabilidades envolvidas passam a ser objetivas; e a problemática da escolha se torna aquela resumida na Proposição de Bernoulli; de modo que um TD maximiza sua utilidade esperada.

Na próxima seção apresenta-se o experimento que deu origem ao fenômeno da ambiguidade na literatura e os modelos subsequentes mais relevantes.

\subsection{O Paradoxo de Ellsberg}

Foi em 1961 que Daniel Ellsberg propôs o experimento que introduziria a ambiguidade na teria da escolha. Motivado pelo ceticismo acerca da distinção entre Risco e Incerteza introduzida por Knight [15]; Ellsberg propõe um questionamento das hipóteses amplamente adotadas no estudo das decisões sob incerteza.

If our pattern of bets were suitably orderly - if it satisfied certain postulated constraints - it would be possible to infer for ourselves numerical subjective probabilities for events, in terms of which some future decisions could be predicted or described. Thus a good deal - perhaps all - of Knight's class of "unmeasurable uncertainties" would have succumbed to measurement, and "risk" would prevail instead of "uncertainty". Ellsberg [6]

Assim, sob os axiomas já propostos na literatura, "for a rational man all uncertainties can be reduced to risk" ${ }^{16}$.

The propounders of these axioms tend to be hopeful that the rules will be commonly satisfied, at least roughly and most of the time, because they regard these postulates as normative maxims, widely acceptable principles of rational behaviour. In other words, people should tend to behave in the postulated fashion, because that is the way they would want to behave. [6].

O autor segue questionando essa abordagem axiomática.

A side effect of the axiomatic approach is that it supplies, at last (as Knight did not), a useful operational meaning to the proposition that people do not always assign, or act "as though" they assigned, probabilities to uncertain events. The meaning would be that with respect to certain events they did not obey, nor did they wish to obey -even on reflection-Savage's postulates or equivalent rules. One could emphasize here either that the postulates failed to be acceptable in those circumstances as normative rules, or that they failed to predict reflective choices; I tend to be more interested in the latter aspect, Savage no doubt in the former.

\footnotetext{
${ }^{16}$ Ellsberg [6]
} 
But from either point of view, it would follow that there would be simply no way to infer meaningful probabilities for those events from their choices, and theories which purported to describe their uncertainty in terms of probabilities would be quite inapplicable in that area.[6].

Ellsberg sugere então o seguinte experimento: Suponha que você é confrontado com duas urnas contendo bolas vermelhas e pretas, e uma bola será retirada de uma delas. Você então é convidado a fazer uma aposta. Apostar em "Vermelho ${ }_{I}$ " significa que você irá retirar uma bola da urna I, se a bola retirada for vermelha você receberá um prêmio $a$ (por exemplo $\mathrm{R} \$ 100,00$ ), se sair uma bola preta você receberá um prêmio $b$ menor (por exemplo $\mathrm{R} \$ 10,00)$. Você sabe que a urna I contém 100 bolas, mas a proporção de bolas pretas e vermelhas é desconhecida. A urna II tem exatamente 50 bolas pretas e 50 vermelhas.

Um observador externo acompanha seu processo de decisão com o objetivo de inferir sua probabilidade subjetiva no processo de escolha. Ele te faz as seguintes perguntas:

1. "Qual aposta você prefere: Vermelho $_{I}$ ou Preto $_{I}$, ou você é indiferente?".Ou seja, ao retirar uma bola da urna I, em qual evento você prefere apostar $\mathrm{R} \$ 100,00$, vermelho ou preto? Ou você não se importa?

2. "Qual aposta você prefere: Vermelho $_{I I}$ ou Preto PII $_{\text {? }}$

3. "Qual aposta você prefere: Vermelho ou Vermelho $o_{I I}$ ?

4. "Qual aposta você prefere: Preto I ou Preto ${ }_{I I}$ ?

Suponha que a sua resposta para o primeiro e segundo itens é "indiferente" 17 . Analisando um grande número de respostas em condições que o autor cita como não-experimentais; Ellsberg considera provável que a sua resposta às duas últimas perguntas se encaixe em dos dos seguintes grupos:

(i) Você é indiferente entre as propostas apresentadas em 3 e 4.

(ii) Você prefere apostar em Vermelho ${ }_{I I}$ ao invés de Vermelho Ve em Preto $_{I I}$ no lugar de Preto $_{I}{ }^{18}$

(iii) Você prefere Vermelho $_{I}$ do que Vermelho $_{I I}$, e Preto $_{I}$ do que Preto $_{I I}{ }^{19}$

Digamos que, por hipótese, você pertence ao grupo (ii). Desse modo, o observador externo irá tentar tirar conlusões a partir das suas escolhas. Ele concluiria então que você considera $V$ ermelho $_{I I}$ como mais provável do que Vermelho $o_{I}$. Mas você também prefere Preto II $_{\text {a }}$ Preto . Note que ele não pode concluir que você considera $V e r m e l h o_{I I}$ como mais provável que

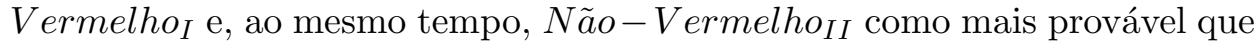
$N \tilde{a} o-V e r m e l h o_{I}$ (já que isto violaria propriedades essenciais das probabilidades); assim a conclusão seria que as suas escolhas simplesmente não revelam julgamentos de probabilidade. Isso acontece porque as suas escolhas violam os axiomas de Savage. ${ }^{20}$

Ellsberg cita outro exemplo que ilustra melhor essa questão. Considere uma urna que contém 30 bolas vermelhas e 60 bolas pretas e amarelas, mas em proporção desconhecida. Você deve retirar uma bola aleatoriamente da urna considerando as seguintes ações:

\footnotetext{
${ }^{17}$ Ellsberg comenta que, de fato, essa é a resposta mais comum.

${ }^{18}$ Esse grupo é citado como maioria.

${ }^{19}$ Esse grupo é citado como minoria nos experimentos informais de Ellsberg.

${ }^{20}$ Mais especificamente, o grupo de escolhas (ii) viola "O Princípio da Coisa Segura".
} 


\begin{tabular}{|c||c|c|c|}
\hline & Vermelha & Preta & Amarela \\
\hline \hline I & $R \$ 100$ & $R \$ 0$ & $R \$ 0$ \\
\hline II & $R \$ 0$ & $R \$ 100$ & $R \$ 0$ \\
\hline
\end{tabular}

A primeira ação é uma "aposta no vermelho"; II uma "aposta no preto". Qual você prefere?

Agora considere as próximas ações sob as mesmas circuntâncias:

\begin{tabular}{|c||c|c|c|}
\hline & Vermelha & Preta & Amarela \\
\hline \hline III & $R \$ 100$ & $R \$ 0$ & $R \$ 100$ \\
\hline IV & $R \$ 0$ & $R \$ 100$ & $R \$ 100$ \\
\hline
\end{tabular}

O ato III é uma "aposta no vermelho ou no amarelo"; IV "aposta no preto ou amarelo". Qual você prefere? O autor descreve então o padrão de resposta mais frequente: o ato I é preferível ao II, e o IV preferível ao III.

No entanto, ao preferir I no lugar de II, revela-se uma preferência pelo vermelho, já que o pay-off de amarelo é constante e, portanto, não serve como critério de desempate (segundo o Princípio da Coisa Segura de Savage). Aplicando o mesmo raciocínio para as opções III e IV, a coluna "Amarelo" continuaria indiferente já que tem pay-off $\mathrm{R} \$ 100,00$ para ambos os atos; assim, aplicando o Princípio da Coisa Segura, a escolha racional seria preferir III ao invés de IV. Porém esse não é o padrão recorrente de respostas. Assim, o postulado P2 de Savage é violado para a maioria das pessoas que participam desse experimento.

Vale mencionar que não seria possível inferir uma probabilidade qualitativa para os eventos baseando-se nas escolhas acima; e mais, não é possível inferir qualquer medida de probabilidade, nem aproximada ou estimada, sob quais as escolhas acima resultariam de um problema de maximização da utilidade esperada.

A teoria até então considerava tais escolhas como irracionais. Ellsberg, no entanto, afirma que não é este o caso em seu experimento; que indivíduos instruídos e definitivamente racionais mantém o padrão de escolha mencionado, mesmo quando lhes é explicado que eles estão violando um dos postulados do modelo UES.

A questão que então se coloca é a seguinte:

Individuals who would choose I over II and IV over III in the example above are simply not acting "as though" they assigned numerical or even qualitative probabilities to the events in question. There are, it turns out, other ways for them to act. But what are they doing? [6].

Ellsberg argumenta que os mesmos indivíduos violadores de P2 em seu experimento, provavelmente não o fariam em outras situações. Assim, ele não procurou desvalidar a teoria da escolha precedente; mas sim introduzir um novo fenômeno informacional que se reflete em diferentes padrões de escolha: a ambiguidade.

Responses from confessed violators indicate that the difference is not to be found in terms of the two factors commonly used to determine a choice situation, the relative desirability of the possible pay-off's and the relative likelihood of the events affecting them, but in a third dimension of the problem of choice: the 
nature of one's information concerning the relative likelihood of events. What is at issue might be called the ambiguity of this information, a quality depending on the amount, type, reliability and "unanimity" of information, and giving rise to one's degree of "confidence" in an estimate of relative likelihoods.[6].

No entanto, quando um agente está disposto a basear suas decisões em uma particular distribuição, os axiomas de Savage e a abordagem Bayesiana se fazem adequadas. As situações que o autor questiona, são aquelas em que os julgamentos de probabilidade são vagos ou pouco confiáveis. Assim, algumas circunstâncias são mais propensas a ambiguidade do que outras. Por exemplo, o lançamento de uma moeda em comparação ao retorno de um novo projeto de investimento por uma empresa.

Assim o fenômeno da ambiguidade surge em situações particulares, em que o conjunto de informações disponíveis é vago; fenômeno esse que os modelos anteriores se mostravam incapazes de incorporar.

"...there seem to be some probability relations about which we feel relatively "sure" as compared with others...The notion of "sure" and "unsure" introduced here is vague, and my complaint is precisely that neither the theory of personal probability, as it is developed in this book, nor any other device known to me renders the notion less vague...A second difficulty, perhaps closely associated with the first one, stems from the vagueness associated with judgements of the magnitude of personal probability". [17].

Com o intuito de incorporar questões até então inexploradas, e motivados pelo Paradoxo de Ellsberg, vários autores sugeriram modelos alternativos capazes de incorporar o fenômeno da ambiguidade na teoria normativa. Afinal, segundo Savage:

"If, after thorough deliberation, anyone maintains a pair of distinct preferences that are in conflict with [a given normative principle] he must abandon, or modify, the principle; for that kind of discrepancy seems intolerable in a normative theory". [17].

\subsection{O Modelo Maxmin de Utilidade Esperada}

Tentando explicar o comportamento do Paradoxo de Ellsberg, Gilboa e Schmeidler (G\&S) publicam em 1989 um modelo importante na teoria da ambiguidade, o Maxmin de Utilidade Esperada (denotado aqui por MMUE) ou Modelo de Múltiplos Antecedentes.

"One conceivable explanation of this phenomenon which we adopt here is as follows: [...] the subject has too little information to form a prior $^{21}$. Hence (s)he considers a set of priors as possible. Being uncertainty averse, (s)he takes into account the minimal expected utility (over all priors in the set) while evaluating a bet". $[12]$.

\footnotetext{
${ }^{21}$ Entende-se prior por medida de probabilidade subjetiva.
} 
G\&S propõe uma fundação axiomática para tal regra de decisão, baseada na estrutura de AA [14]. Como a intenção é captar ambiguidade, os autores optaram por enfraquecer o axioma da independência ${ }^{22}$, o substituindo por dois outros.

De maneira geral, MMUE estende a abordagem bayesiana (com diferente interpretação) para o caso de múltiplos priors no lugar de uma única medida. Para tanto, é introduzido o conjunto $C^{23}$ de todas as possíveis distribuições de probabilidade em um problema de decisão estatístico.

Os autores mantém os axiomas de Ordem Fraca, Monotonicidade, Continuidade e Não-Degeneração. A independência é substituída por:

- C-independência: Para quaisquer $f, g$ e qualquer ato constante $h$, $\forall \alpha \in(0,1): f \succsim g \Leftrightarrow \alpha f+(1-\alpha) h \succsim \alpha g+(1-\alpha) h$.

- Aversão à Incertezas: Para todo $f, g$ e $\alpha \in(0,1): f \sim g \Rightarrow$ $\alpha f+(1-\alpha) g \succsim g$.

Note que o axioma da independência no escopo AA é mais forte do que a C-independência aqui considerada, já que antes $h$ poderia ser qualquer ato e aqui tal função é considerada constante.

Gilboa e Schmeidler, através de seu teorema de representação, demonstram que os indivíduos que satisfazem os axiomas acima podem ter suas escolhas representadas pelo funcional: ${ }^{24}$

$$
W(f(\cdot))=\min _{\mu \in C} \int U(f(\cdot)) d \mu
$$

Sendof $f(\cdot)$ o ato em consideração, $C$ o conjunto de priors sobre $S$ e $U(\cdot)$ a função utilidade do tipo vN-M.

\subsection{O Modelo de Preferências Variacionais}

Maccheroni, Marinacci e Rustichini propõe em 2006 o Modelo de Preferências Variacionais (MPV) que conseguiu generalizar dois modelos de escolha sob ambiguidade importantes: O maxmin visto na seção anterior, e o Multiplier Preferences (MP).

A idéia central é a mesma do MMUE: um indivíduo frente à ambiguidade não considera uma única medida de probabilidade, mas sim um conjunto delas. A diferença agora é que os autores propõe um índice de ambiguidade, definido sobre o conjunto das medidas, que captaria a atitude ambígua.

Já em MP, o agente leva em consideração que o seu modelo pode não ser o correto, mas apenas uma aproximação; através do funcional de escolha:

$$
V(f)=\min _{p \in \Delta(S)}\left\{\int u(f) d p+\theta R\left(\mu \| \mu^{*}\right)\right\}
$$

\footnotetext{
${ }^{22}$ Normalmente todos os modelos de ambiguidade enfraquecem independência; isso porque o Paradoxo de Ellsberg mostra um caso de violação do Princípio da Coisa Segura. Mais adiante veremos o modelo Bewley [2] que opta pelo enfraquecimento da completude.

${ }^{23}$ Considera-se que o conjunto $C$ é convexo e fechado.

${ }^{24}$ Vale mencionar que a topologia considerada em MMUE sobre o espaço de funções finitamente aditivas em $\Sigma$ é a topologiafraca* ${ }^{*}$ Lembrando que o conjunto das medidas de probabilidade finitamente aditivas sob esta topologia é compacto. Veja, por exemplo, $[3]$.
} 
sendo $\theta \geq 0$ um parâmetro, e $R\left(\mu \| \mu^{*}\right): X \rightarrow[0, \infty]$ a entropia relativa do prior $\mu$ com respeito ao prior de referência $\mu^{* 25}$; sendo essa uma medida de "distância" entre duas medidas de probabilidade: $\mu$ e $\mu^{*}$. Desse modo:

Agents who behave according to this choice criterion are considering the possibility that $\mu$ may not be the appropriate law that governs the phenomenon in which they are interested and for this reason they take into account other possible models $\mu^{*}$. The relative likelihood of these alternative models is measured by their relative entropy, while the positive parameter $\theta$ reflects the weight that agents are giving to the possibility that $\mu^{*}$ may not be the correct model. As the parameter $\theta$ becomes larger, agents focus more on $\mu^{*}$ as the correct model, giving less importance to possible alternative models $\mu$.

A partir disso, define-se um funcional de escolha que engloba MMUE e Multiplier Preferences como casos particulares; para tanto os autores utilizam os mesmos axiomas do modelo Maxmin com uma versão enfraquecida da C-independência.

- C-Independência Fraca: $\forall f, g \in \mathcal{F} ; \forall x, y \in X ; \forall \alpha \in(0,1)$; se $\alpha f+(1-\alpha) x \succsim \alpha g+(1-\alpha) x$, então $\alpha f+(1-\alpha) y \succsim \alpha g+(1-\alpha) y$.

Os autores demonstram que os axiomas de Ordem Fraca, Não-Degeneração, Monotonicidade, Continuidade, Aversão à Incerteza e C-independência Fraca são necessários e suficientes para a existência da seguinte representação:

$$
V(f)=\min _{p \in \Delta(S)}\left\{\int u(f) d p+c(p)\right\}
$$

Sendo $c: X \rightarrow[0, \infty]$ uma função convexa semicontínua inferior, chamada função custo, que satisfaz $\min _{\mu \in \Delta(S)} c(\mu)=0$.

A função custo $c$ é o ponto central da representação, sendo através dela que os modelos MMUE e Multiplier Preferences podem ser obtidos. Ao fixar a utilidade, a função custo fica também unicamente definida.

Os autores demonstram então como obter o Modelo Maxmin através do funcional (5.5). Fixa-se um conjunto convexo e compacto $C$ de priors e defini-se:

$$
c(\mu)= \begin{cases}0, & \mu \in C \\ \infty, & \mu \notin C\end{cases}
$$

Com esta especificação (também chamada de "função indicadora") da função custo, fica claro que uma medida $\mu \notin C$ nunca será solução do problema de minimização em (5.5). Note que o custo das medidas pertencentes ao conjunto $C$ é nulo, reduzindo o problema em 5.5 ao caso MMUE.

\footnotetext{
${ }^{25}$ Definimos a entropia relativa com respeito a $\mu^{*}$ por: $R\left(\begin{array}{llll}\mu & \left.\mu^{*}\right)=\end{array}\right.$ $\left\{\begin{array}{l}\int_{S}\left(\log \frac{d \mu}{d \mu^{*}}\right) d \mu, \quad \text { se } \quad \mu \in X \\ \infty \quad \text { c.c. }\end{array}\right.$
} 


\subsection{O Modelo Bewley de Preferências Incompletas}

Diferentemente dos outros modelos de ambiguidade, Bewley propõe uma fundação axiomática sem completude (os outros modelos enfraqueciam a independência). O autor assume que, em situações ambíguas, indivíduos simplesmente não seriam capazes de comparar alguns atos entre si.

Bewley utiliza o escopo AA e estipula uma regra de uninamidade que agentes utilizariam na decisão.

The basic idea is to drop the completeness assumption from Savage's [17] framework and to add an assumption of inertia. The completeness assumption asserts that any two lotteries are comparable in the preference ordering; that is, one is preferred to the other or they are equivalent in the ordering. When this axiom is dropped, one obtains a set of subjective probability distributions rather than a single one. One lottery is preferred to another if its expected value is higher according to all the distributions. The idea of the inertia assumption is that a person never accepts a lottery unless he prefers acceptance to rejection. There is a status quo with which he stays unless a alternative is preferred. Without the inertia assumption, choices between all incomparable alternatives would be arbitrary. [2].

Em seu modelo, Bewley enfraquece o axioma AA.1 da Ordem Fraca e o substitui por dois outros:

- Ordem Parcial: $\succsim \in \mathcal{F}$ é reflexiva e transitiva.

- C-Completude: Para todo ato constante $x$ e $y$, tem-se $x \succsim y$ ou $y \succsim x$.

O axioma da C-completude mostra que o TD conhece suas preferências; assim, quando probabilidades objetivas estão em jogo ele sabe comparar entre dois atos. Dessa forma, incompletude se torna uma consequência da ignorância sobre as probabilidades.

Também adota-se a suposição de inércia: O TD permanece no status quo a não ser que exista uma alternativa preferível segundo todas as distribuições de probabilidade. Assim, frente a urna de Ellsberg, um tomador de decisão segundo Bewley, se declararia incapaz de escolher entre os atos a sua disposição optando pela inércia.

De acordo com este modelo, para quaisquer dois atos $f$ e $g$, tem-se $f$ fracamente preferível a $g$ se e somente se:

$$
\int u(f(\cdot)) d \mu \geq \int u(g(\cdot)) d \mu \quad \forall \mu \in C
$$




\section{Capítulo 6}

\section{Preferências Bewley Variacionais}

Apresenta-se agora o modelo de principal interesse no presente trabalho; nele Faro [8] conseguiu generalizar importantes modelos da teoria da ambiguidade.

A motivação continua a mesma; ou seja, estudar situações econômicas em que indivíduos são incapazes de obter um ranking de alternativas devido ao estado informacional em que se encontram. Uma manifestação desse estado informacional de ambiguidade são as preferências incompletas conforme discutido por Bewley [2].

The Knightian decision theory, as proposed by Bewley [2], ${ }^{1}$ characterizes DMs with incomplete preferences due to the multiple priors nature of beliefs.

The Knightian decision theory describes a DM that holds a set of beliefs $C$ and follows a unanimity rule given by a priorby-prior dominance: an act $f$ is at least as desirable as $g$ if, and only if, the expected utility of $f$ is not less than the expected utility of $g$, for all priors in $C$.

Faro [8] segue com uma possível interpretação do conjunto $C$ :

A natural interpretation for Bewley's preferences considers a DM as coping with many opinions revealed by experts or specialists. (...) The set of multiple priors $C$ may capture the universe of all opinions defended by some Bayesian expert, and one act is weakly preferred over another if its associated expected utility exceeds or equals that of the alternative according to every expert's opinion in the set. An important feature of this unanimity rule is that every opinion is treated with the same weight. In view of this, it is natural to wonder how restrictive is such uniform weighting?

Com essa preocupação em mente, Faro [8] propõe uma regra de decisão em que o TD atribui pesos distintos às diversas opiniões à sua disposição.

Let us consider a policy maker that wants to take a decision on a given subject, with the support of some leading economists. Since

\footnotetext{
${ }^{1}$ É possível interpretar a contribuição de Bewley [2] como uma possível formalização da teoria discutida por Knight [15].
} 
experts usually have different priors on scenarios, the policy maker likely faces the problem of a disagreement on the ranking of certain pairs of acts. For instance, we may think of the chairperson of the Fed asking economists for advice regarding monetary policy. Decisions made by monetary authority are subjective regarding future states of the economy. Assume that between two available acts $f$ and $g$ some economists (group A) claim that the net expected utility $E[u(f)-u(g)]$ is positive and high (a substantial gain), while other economists (group B) disagree and claim that the corresponding net expected utility is negative and close to zero (a small loss). Following Bewley's unanimity rule the $D M$ should not switch from $g$ to $f$. We are concerned here with this restrictive feature of the unanimity rule, and the purpose of this paper is to present a more flexible dominance criterion, in the sense that a DM might take into account different pieces of advice with different weights. [8].

Desse modo, o objetivo central do autor é fornecer uma regra de decisão em que a confiança do tomador de decisão possa variar entre opiniões. O que se traduziria no exemplo acima com o presidente do Fed atribuindo um peso maior à opinião dos economistas do grupo A do que do grupo $\mathrm{B}$.

Para tanto, será necessário um enunciado de axiomas mais relaxado do que o proposto por Bewley [2]. Isso porque tal regra de decisão pode violar (além da independência), transitividade.

Faro [8] demonstra essa situação utilizando o mesmo exemplo do Fed: Considere três opções $f, g$ e $h$ em que qualquer ranking entre elas seja possível; segundo a regra de uninamidade, o presidente do Fed não deveria adotar nenhuma delas e permanecer no status quo. Porém suponhamos que, para o presidente do Fed, a opinião de alguns tem mais relevância do que a de outros.

Considere que o grupo de economistas mais respeitado concorde entre si que $f$ é bem melhor do que $g$, e $g$ é bem melhor do que $h$. Por outro lado, o grupo menos relevante considera $g$ fracamente melhor do que $f$ e $h$ é fracamente melhor do que $g$. Após considerar ambos, suponha que o TD decide seguir a opinião do primeiro grupo e revela a preferência $f \succsim g \mathrm{e}$ $g \succsim h$. No entanto, o TD poderia se mostrar incapaz de comparar $f$ e $h^{2}$, o que contraria o axioma usual da transitividade.

\subsection{Escopo do Modelo}

O autor segue dentro do escopo Anscombe e Aumann apresentado na seção 5.2 .

Considere o conjunto $S$ de estados da natureza, dotado de uma $\sigma$-algebra $\Sigma$ de subconjuntos chamados eventos, e um conjunto não vazio $X$ de consequências. Denota-se por $\mathcal{F}$ o conjunto de todos os atos: funções finitas ${ }^{3}$ do tipo $f: S \rightarrow X, \Sigma$-mensuráveis.

Denota-se por $B_{0}(\Sigma)$ o conjunto de todas as funções reais finitas simples, $\Sigma$-mensuráveis do tipo: $a: S \rightarrow \mathbb{R}$. A norma em $B_{0}(\Sigma)$ é dada por $\|a\|_{\infty}=$

\footnotetext{
${ }^{2}$ No cenário apresentado, uma possibilidade seria os especialistas mais confiáveis concluírem que $f$ é muito melhor do que $h$ e os menos confiáveis $h$ melhor do que $f$, deixando o presidente sem comparação entre $f$ e $h$.

${ }^{3}$ Uma função real é finita quando o seu conjunto imagem é limitado.
} 
$\sup _{s \in S}|a(s)|$ (chamada de norma do sup); $B(\Sigma)$ denota o fecho pela norma do sup de $B_{0}(\Sigma)$. O autor ressalta que $B_{0}(\Sigma)$ pode também ser interpretado como o espaço vetorial gerado pelas funções indicadoras dos elementos de $\Sigma$, dotado da norma do $\sup ^{4}$.

Seja $b a(\Sigma)$ o espaço de Banach de todas as funções finitas e aditivas definidas em $\Sigma$ dotadas da norma da variação total; que é isometricamente isomorfo à norma do dual de $B_{0}(\Sigma)$. Note que a topologia fraca* $\sigma\left(b a, B_{0}\right)$ de $b a(\Sigma)$ coincide com a topologia da convergência pontual. Assume-se que qualquer subconjunto de $b a(\Sigma)$ é dotado da topologia inerente da topologia fraca*.

Dado o mapa $u: X \rightarrow \mathbb{R}$, a função $u(f): S \rightarrow \mathbb{R}$ fica definida por $u(f)(s)=u(f(s))$ para todo $s \in S$. Note que $u(f) \in B_{0}(S, \Sigma)$ sempre que $f \in \mathcal{F}$. Se $x \in X$, define-se $x \in \mathcal{F}$ como o ato constante tal que $x(s)=x$ para todo $s \in S$. Desse modo, $f(s) \in X$ também representa o ato constante que retorna $f(s)$ em todo estado da natureza.

Assume-se que o conjunto $X$ de consequências é um subconjunto convexo de um espaço vetorial ${ }^{5}$. Assim é possível definir para todo $f, g \in \mathcal{F}$ e $\alpha \in[0,1]$ o ato $\alpha f+(1-\alpha) g: S \rightarrow X$ como $(\alpha f+(1-\alpha) g)(s)=\alpha f(s)+$ $(1-\alpha) g(s), \forall s \in S$. Também, considerando dois atos $f, g \in \mathcal{F}$ e um evento $E \in \Sigma$, denotamos por $f E g$ o ato que retorna $f(s)$ em $E$ e $g(s)$ em $E^{c}$.

Seja $\Delta:=\Delta(\Sigma)$ o conjunto de todas as medidas de probabilidade (finitamente aditivas) $p: \Sigma \rightarrow[0,1]$, dotado da topologia inerente da topologia fraca*.

Diz-se que um mapa $\eta: \Delta \rightarrow[0, \infty]$ é grounded se $\{\eta=0\}:=\{p \in$ $\Delta: \eta(p)=0\} \neq \emptyset$. Sendo o domínio de $\eta: \Delta \rightarrow[0, \infty]$ definido como $\{\eta<\infty\}:=\{p \in \Delta: \eta(p)<+\infty\} ;$ e $\overline{\{\eta<\infty\}}$ denota seu fecho. Lembramos que, $\eta$ é semicontínua inferior se $\{\eta \leq r\}$ for fechado para cada $r \geq 0$. Considera-se $\mathcal{N}(\Delta)$ a classe de todas as funções $\eta$ convexas, semicontínuas inferiores e grounded.

\subsection{Axiomas}

As preferências do tomador de decisão são representadas por uma relação binária $\succsim$ em $\mathcal{F}$, cujos componentes simétricos e assimétricos ficam representados por $\sim \mathrm{e} \succ$.

Seguem os axiomas da relação de preferência $\succsim:$

Axioma 1: Reflexividade: Para todo $f \in \mathcal{F}, f \succsim f$.

Axioma 2: Transitividade sem ambiguidade: Suponha $f \succsim g$. Se $h(s) \succsim$ $f(s)$ para todo $s$, então $h \succsim g$. Também, se $g(s) \succsim h(s)$ para todo $s$, então $f \succsim h$.

Axioma 3: $C$-Completude: Para todo ato constante $x$ e $y$, tem-se $x \succsim y$ ou $y \succsim x$.

Axioma 4: Continuidade com respeito a misturas: Para todo ato $f, g, h \in$ $\mathcal{F}$ os conjuntos

$$
\{\alpha \in[0,1]: \alpha f+(1-\alpha) g \succsim h\} \quad e \quad\{\alpha \in[0,1]: h \succsim \alpha f+(1-\alpha) g\}
$$

são fechados em $[0,1]$.

Axioma 5: Independência por Dominância: Para todo $f, g, h_{1}, h_{2} \in \mathcal{F}$, e todo $\alpha \in(0,1)$; se $f \succsim g$ e $h_{1} \succsim h_{2}$ então $\alpha f+(1-\alpha) h_{1} \succsim \alpha g+(1-\alpha) h_{2}$.

\footnotetext{
${ }^{4}$ Para mais detalhes veja Dunford e Schwartz [5] Cap. 4 seção 5.

${ }^{5}$ Como, por exemplo, o conjunto de todas as loterias simples sobre um espaço de alternativas $Z$; que é o caso clássico apresentado no escopo AA.
} 
Axioma 6: Não limitação sobre as loterias: Existem $x, y \in X$ tais que para cada $\alpha \in(0,1)$, existem $z, \hat{z} \in X$ tais que

$$
\alpha z+(1-\alpha) y \succ x \succ y \succ \alpha \hat{z}+(1-\alpha) x
$$

Analisando os axiomas propostos temos:

- Reflexividade: basicamente garante que um ato $f$ é tão bom quanto ele mesmo.

- Transitividade sem ambiguidade: Como já visto, segundo o modelo PBV o axioma tradicional de transitividade pode ser violado. Assim, o autor sugere uma versão mais branda em seu segundo axioma, o qual enuncia que a transitividade é válida para atos em que a preferência se mantém em todos os pontos (estados da natureza).

- C-Completude: Como uma das premissas do trabalho é que informações assimétricas se manifestam nas escolhas través de preferências incompletas; o axioma 3 enuncia que as relações de preferência $\succsim$ serão completas somente na comparação entre atos constantes.

- Continuidade com respeito a misturas: Uma suposição usual em modelos que utilizam o escopo Anscombe e Aumman [14] (apresentado na seção 5.2).

- Independência Dominada: Como estamos lidando com ambiguidade se faz necessário enfraquecer a independência. Faro [8] adota o axioma 5 afirmando que, misturas de atos preferíveis, devem ser preferidas a misturas (com igual ponderação) de atos dominados.

- Não limitação sobre as loterias: O axioma 6 é uma suposição técnica que garante o mapeamento completo dos reais.

Dizemos que uma relação binária que satisfaz os axiomas 1-6 é do tipo Bewley Variacional (daqui em diante referidas como BV).

\subsection{Representação do Modelo Principal}

Teorema 6.3.1. Seja $\succsim$ uma relação de preferência no conjunto de atos do tipo Anscombe-Aumann $\mathcal{F}$. As seguintes condições são equivalentes:

(1) $\succsim$ satisfaz os axiomas 1-6.

(2) Existe um indice afim de utilidade $u: X \rightarrow \mathbb{R}$, com $u(X)=\mathbb{R}$, e uma função $\eta: \Delta \rightarrow[0, \infty]$ pertencente a $\mathcal{N}(\Delta)$ tal que para todo $f$ e $g \mathrm{em}$ $\mathcal{F}$

$$
f \succsim g \Leftrightarrow \int u(f) d p+\eta(p) \geq \int u(g) d p, \forall p \in \Delta
$$

Para cada u existe uma única $\eta^{*}: \Delta \rightarrow[0, \infty]$ consistente com a representação acima dada por

$$
\eta^{*}(p)=\sup \left\{\int(u(g)-u(f)) d p: f \succsim g\right\}, \forall p \in \Delta
$$


Podemos interpretar (6.1) como uma regra de decisão unânime ponderada, na qual a função $\eta$ representa o peso dado a cada prior; sendo que maiores valores de $\eta$ correspondem a priors com menor peso. Além disso, $\eta^{*}(p)$ pode ser considerada a perda líquida máxima aceita pelo TD quando considera a prior $p$.

Assim, o Teorema 6.3.1 afirma que $f \succsim g$ se, e somente se, para todo $p \in \Delta$, a utilidade esperada líquida $\int(u(f)-u(g)) d p$ não gera um sacrifício maior do que a perda dada por $\eta(p)$; ou seja, $\int(u(f)-u(g)) d p \geq-\eta^{*}(p)$ para todo $p \in \Delta$.

\subsection{Extensões a partir de PBV}

Uma grande contribuição foi a possibilidade de obter outros modelos de preferências ambíguas a partir de PBV ao acrescentarmos algumas hipóteses sobre os axiomas.

Ao completar a relação de preferências do indivíduo, PBV se torna equivalente ao UES. Se esse completamento for fraco, o autor demonstra que PBV chega no caso de Preferências Variacionais; que por sua vez tem MMUE como caso particular como já apresentado na seção 5.5. Outra equivalência pode ser obtida ao assumir transitividade, conforme explicaremos na próxima seção.

\subsubsection{Preferências Bewley Incompletas}

No modelo proposto por Bewley [2] e apresentado na seção 5.6 uma característica adotada é a transitividade, que foi relaxada no teorema principal de PBV. Assim, o teorema abaixo enuncia que, preferências Bewley Variacionais, ao assumirem transitividade, resultam na classe de preferências propostas por Bewley [2] ${ }^{6}$.

Teorema 6.4.1. Seja $\succsim$ uma relação de preferência Bewley Variacional representada por $\left(u, \eta^{*}\right)$. São equivalentes:

(i) A preferência $\succsim$ satisfaz transitividade;

(ii) A preferência $\succsim$ satisfaz independência;

(iii) Para todo $f, g \in \mathcal{F}$

$$
f \succsim g \Leftrightarrow \int u(f) d p \geq \int u(g) d p, \forall p \in\left\{\eta^{*}=0\right\}
$$

(iv) A função $\eta^{*}$ toma somente os valores 0 e $\infty$.

\subsubsection{Preferências Bewley Variacionais Completas}

Vimos que ao satisfazer transitividade (e/ou independência), a classe de preferências BV se reduz às preferências incompletas de Bewley [2]. No entanto, o que aconteceria se assumíssemos completude das preferências no modelo VBP?

Em nosso modelo de interesse, completude resulta em transitividade, que por sua vez resulta na independência. Desse modo obtemos a representação proposta por Anscombe e Aumann [14] de utilidade subjetiva esperada.

\footnotetext{
${ }^{6}$ Demonstração em Faro [8].
} 
Teorema 6.4.2. Se uma preferência Bewley Variacional $\succsim$ representada por $\left(u, \eta^{*}\right)$ é completa, então ela é transitiva. Em particular, ¿é uma preferência de utilidade esperada, conforme aquela apresentada no Teorema (5.2).

\subsubsection{Conexão com Preferências Variacionais}

Uma das manifestações da ambiguidade, como já discutido, é através de preferências incompletas. Porém, em muitos casos, o TD deveria ser capaz de comparar quaiquer dois atos. Assim, o autor sugere uma extensão fraca cuidadosa das preferências BV com o intuito de obter uma relação completa e transitiva.

É através do trabalho GMMS [10] que tal extensão fica bem definida, nele o TD começa com uma relação de preferência incompleta ${ }^{*}$, quando um comportamento do tipo maxmin emerge como possível complemento de ‡*. Baseando-se nesse raciocício, Faro [8] consegue tal extensão partindo de preferências BV.

Denota-se $\succsim^{* *}$ pela extensão fraca de $\succsim^{*}$; que, por hipótese, é uma préordem contínua e monótona. Definimos mais alguns axiomas necessários: Sejam $f, g \in \mathcal{F}$ e $x \in X$,

- Consistência Fraca ${ }^{7}: f \succsim^{*} x$ implica $f \succsim^{* *} x$.

- Default à certeza: Se não $f \succsim^{*} x$, então $x \succ^{* *} f$.

A consistência fraca afirma que $\succsim^{* *}$ mantém a relação de preferências de ${ }^{*}$ quando compara-se um ato $f \in \mathcal{F}$ com o ato constante $x \in X$. Já o default a certeza captura aversão à ambiguidade; isso porque se não houverem razões para o TD escolher $f$ ao invés de $x$, então ele prefere estritamente $x$ (que tem risco mas não incerteza), ao invés do ato incerto $f$.

O resultado seguinte obtém preferências variacionais (MMR [16]) como uma extensão fraca de preferências Bewley Variacionais. Para tanto, é necessário impor condições nas relações das preferências $\succsim^{*} \mathrm{e} \succsim^{* *}$

Teorema 6.4.3. Seja ${ }^{*}$ uma preferência Bewley Variacional representada pelo par $\left(u, \eta^{*}\right)$, e suponha que $\succsim^{* *}$ é uma pré-ordem completa, monótona e contínua. Se $\succsim^{*} e \succsim^{* *}$ satisfazem, conjuntamente, Consistência Fraca e Default à Certeza, então

$$
f \succsim^{* *} g \Leftrightarrow \min _{p \in \Delta} \int u(f) d p+\eta^{*}(p) \geq \min _{p \in \Delta} \int u(g) d p+\eta^{*}(p)
$$

Vale lembrar que, definindo $\eta^{*}(p)$ como em (5.6), consegue-se uma preferência do tipo MMUE . Mais um caso particular que conseguimos a partir do modelo PBV.

\footnotetext{
${ }^{7}$ Em GMMS [9] é adotada uma versão mais forte desse axioma, a consistência: $f \succsim^{*} g$ implica $f \succsim^{* *} g$
} 


\section{Capítulo 7}

\section{Independência e Preferências Bewley Variacionais}

As seções anteriores procuraram salientar a contribuição feita por Faro [8] ao definir uma classe de preferências clara, aplicável e que abrange modelos importantes de ambiguidade. No entanto, o autor inicia uma discussão sobre o axioma da independência em preferências BV que deixa ${ }^{1}$, de certa forma, uma pergunta em aberto: O que as preferências do tipo BV "perdem" do axioma da independência? E o que de fato permanece?

Assim, contribuir para um melhor entendimento do modelo PBV nesse sentido, se tornou o objetivo deste trabalho.

Vamos agora à nossa contribuição ao modelo. Para tanto introduzimos as seguintes definições:

Definição 7.0.1. Independência para pesos constantes (IPC): Para todo $f, g, h_{1}, h_{2} \in \mathcal{F}$, e todo $\alpha \in(0,1)$ :

$$
\alpha f+(1-\alpha) h_{1} \succsim \alpha g+(1-\alpha) h_{1} \Rightarrow \alpha f+(1-\alpha) h_{2} \succsim \alpha g+(1-\alpha) h_{2}
$$

Ou seja, a preferência se mantém quando $h_{1}$ é substituído por $h_{2}$ desde que $\alpha$ seja mantido constante.

Definição 7.0.2. Independência com relação aos pesos (IP): Para todo $f, g, h \in \mathcal{F}$ e todo $\alpha, \beta \in(0,1)$ :

$$
\alpha f+(1-\alpha) h \succsim \alpha g+(1-\alpha) h \Rightarrow \beta f+(1-\beta) h \succsim \beta g+(1-\beta) h
$$

Já nesse caso a preferência se mantém quando modificam-se os pesos (troca-se $\alpha$ por $\beta$ ) mas os atos combinados se mantém.

Note que preferências BV satisfazem IPC como demonstrado a seguir.

Proposição 7.0.1. Toda preferência Bewley Variacional satisfaz Independência para Pesos Constantes.

\footnotetext{
${ }^{1}$ Veja [8] pg. 706.
} 
Demonstração. Sejam $f, g, h_{1} \in \mathcal{F}$ tais que

$$
\alpha f+(1-\alpha) h_{1} \succsim \alpha g+(1-\alpha) h_{1}
$$

sendo $\succsim$ uma preferência do tipo Bewley Variacional e $\alpha \in(0,1)$.

Pelo Teorema de Representação das preferências BV segue que:

$$
\int\left(\alpha u(f)+(1-\alpha) u\left(h_{1}\right)\right) d p+\eta(p) \geq \int\left(\alpha u(g)+(1-\alpha) u\left(h_{1}\right)\right) d p, \forall p \in \Delta
$$

Note que

$$
(7.1) \Leftrightarrow \alpha \int u(f) d p+\eta(p) \geq \alpha \int u(g) d p
$$

Agora considere $h_{2} \in \mathcal{F}$.

Desse modo

$(7.2) \Leftrightarrow \alpha \int u(f) d p+(1-\alpha) \int u\left(h_{2}\right) d p+\eta^{\prime}(p) \geq \alpha \int u(g) d p+(1-\alpha) \int u\left(h_{2}\right) d p$

Logo

$$
\begin{aligned}
(7.3) & \Leftrightarrow \int\left[\alpha u(f)+(1-\alpha) u\left(h_{2}\right)\right] d p+\eta^{\prime}(p) \geq \int\left[\alpha u(g)+(1-\alpha) u\left(h_{2}\right)\right] d p \\
& \Leftrightarrow \alpha f+(1-\alpha) h_{2} \succsim \alpha g+(1-\alpha) h_{2}
\end{aligned}
$$

Desse modo, $\forall f, g, h_{1} \in \mathcal{F}$ e $\alpha \in(0,1)$ temos

$$
\alpha f(1-\alpha) h_{1} \succsim \alpha g+(1-\alpha) h_{1} \Rightarrow \alpha f+(1-\alpha) h_{2} \succsim \alpha g+(1-\alpha) h_{2}
$$

Vamos agora enunciar um lema que será muito útil mais adiante:

Lema 7.0.3. Uma relação binária $\succsim$ em $\mathcal{F}$ satisfaz independência se, e somente se, para $f, g, h_{1}, h_{2} \in \mathcal{F}$ e $\alpha, \beta \in(0,1]$

$$
\alpha f+(1-\alpha) h_{1} \succsim \alpha g+(1-\alpha) h_{1} \Rightarrow \beta f+(1-\beta) h_{2} \succsim \beta g+(1-\beta) h_{2}
$$

De outro modo, uma relação binária $\succsim$ em $\mathcal{F}$ satisfaz independência, se e somente se, satisfaz IPC e IP.

Demonstração. Suponha que $\succsim$ satisfaça independência. Tomemos $f, g, h_{1}, h_{2}, h \in$ $\mathcal{F}$ e $\alpha, \beta \in(0,1]$.

Vamos fazer a ida: considere

$$
\alpha f+(1-\alpha) h_{1} \succsim \alpha g+(1-\alpha) h_{1}
$$

Por independência segue que $f \succsim g$.

E por independência novamente temos $\beta f+(1-\beta) h_{2} \succsim \beta g+(1-\beta) h_{2}$.

Desse modo temos: 


$$
\alpha f+(1-\alpha) h_{1} \succsim \alpha g+(1-\alpha) h_{1} \Rightarrow \beta f+(1-\beta) h_{2} \succsim \beta g+(1-\beta) h_{2}
$$

Agora vamos provar a volta. Podemos escrever que

$$
f \succsim g \Rightarrow \beta f+(1-\beta) h \succsim \beta g+(1-\beta) h
$$

Agora se $\beta f+(1-\beta) h \succsim \beta g+(1-\beta) h$,

É possível obter, para $\beta=1$, que $f \succsim g$.

Assim, vale a independência.

Em outras palavras, a classe de preferências Bewley Variacionais permite revezes de preferência na mistura de atos a menos que os pesos de tal mistura sejam mantidos constantes. Mais adiante veremos que IP é um modo de obter transitividade das preferências BV.

Nessa seção buscamos definir outra maneira de obter preferências a la Bewley a partir de preferências BV. Já vimos que Independência é equivalente a Transitividade sob Independência Dominada; ou seja, toda preferência BV que satisfaça Independência pode ser representada pela regra de decisão unânime de Bewley.

Além disso, vimos que Independência é equivalente a IPC + IP. Assim, usando (7.0.1), temos que uma preferência BV $\succsim$ satisfaz IP se, e somente se, $\succsim$ for uma preferência a la Bewley. Dessa forma,

Teorema 7.0.4. Seja $\succsim$ uma preferência Bewley Variacional representada por $\left(u, \eta^{*}\right)$. São equivalentes:

(i) A preferência $\succsim$ satisfaz Independência com relação aos pesos;

(ii) Para todo $f, g \in \mathcal{F}$

$$
f \succsim g \Leftrightarrow \int u(f) d p \geq \int u(g) d p, \forall p \in\left\{\eta^{*}=0\right\}
$$

Demonstração. (i) $\Rightarrow$ (ii): Pela Proposição (7.0.1) sabemos que toda preferência BV satisfaz IPC; no entanto, ao satisfazer IP, pelo lema (7.0.3), resulta que as preferências também satisfazem o axioma da independência.

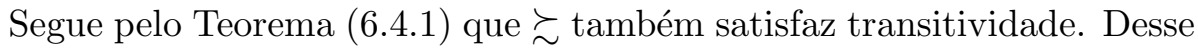
modo, os axiomas se reduzem ao caso Bewley [2], sendo possível obter uma regra unânime de decisão como a apresentada em (ii).

(ii) $\Rightarrow$ (i): A regra unânime de decisão se aplica no caso das preferências incompletas a la Bewley [2], que satisfazem independência. Pelo lema (7.0.3) segue (i). 


\section{Capítulo 8}

\section{Conclusão}

Na seção anterior dividimos o axioma da independência em dois outros: a independência quando os pesos são mantidos constantes (IPC) e quando não são (IP). Essa abordagem permite responder a pergunta inicial: O que as preferências do tipo BV "perdem" do axioma da independência? E o que de fato permanece?

Como foi demonstrado que toda preferência BV é independente quando os pesos são mantidos constantes (IPC), tem-se aí a resposta. Ou seja, o modelo Bewley Variacional, ao incorporar ambiguidade; "perde" o tipo de independência que permite a troca dos pesos $\alpha$ e $\beta$ (IP).

Assim, concluí-se que o presente trabalho contribui para um melhor entendimento das preferências Bewley Variacionais com respeito à independência. Porém, também deixou uma questão aberta: PBV satisfazem IPC mas não IP; existiriam classes de preferência que satisfazem IP e não IPC? 


\section{Apêndice A}

\section{Apêndice}

\section{A.1 Demonstrações PBV}

\section{A.1.1 Relações Binárias Fundamentais}

Seja $\unrhd$ em $B_{0}(\Sigma)$ uma relação binária que assumimos não-trivial (ou seja, existem $a$ e $b \in B_{0}(\Sigma)$ tais que $a \triangleright b$; sendo $\triangleright$ a parte assimétrica de $\unrhd$ ). A seguir definimos algumas propriedades fundamentais:

- $\unrhd$ é Reflexiva se $a \unrhd a$ para todo $a \in B_{0}(\Sigma)$.

- $\unrhd$ satisfaz Transitividade sem ambiguidade se dados $a, b, c \in B_{0}(\Sigma)$ tais que $a \unrhd b$; se $c \geq a$ tem-se $c \unrhd b$. E se $b \geq c$, então $a \unrhd c$.

- $\unrhd$ é Transitiva quando para $a, b, c \in B_{0}(\Sigma)$, se $a \unrhd b$ e $b \unrhd c$ tem-se $a \unrhd c$.

- $\unrhd$ é $N a \tilde{o}$-Trivial se existem $a, b \in B_{0}(\Sigma)$ tais que $a \triangleright b$; ou seja, $a \triangleright b$ mas não $b \triangleright a$.

- $\unrhd$ é Contínua se, dada qualquer sequência $\left\{\left(a_{n}, b_{n}\right)\right\}_{n \in \mathbb{N}}$ tais que para todo $n \geq 1,\left(a_{n}, b_{n}\right) \in \unrhd$ se $a_{n} \stackrel{\|\cdot\|_{\infty}}{\longrightarrow} a \in B_{0}(\Sigma)$ e $b_{n} \stackrel{\|\cdot\|_{\infty}}{\longrightarrow} b \in B_{0}(\Sigma)$; então $(a, b) \in \unrhd$. Em outras palavras, $\unrhd$ é um subconjunto fechado de $B_{0}(\Sigma) X B_{0}(\Sigma)$.

- $\unrhd$ é Contínua com respeito a misturas se, para todo $a, b, c \in B_{0}(\Sigma)$, os conjuntos $\{\alpha \in[0,1]: \alpha a+(1-\alpha) b \unrhd c\}$ e $\{\alpha \in[0,1]: c \unrhd \alpha a+(1-\alpha) b\}$ forem fechados em $[0,1]$.

- $\unrhd$ é Afim se para todo $a, b, c \in B_{0}(\Sigma)$ e $\alpha \in(0,1), a \unrhd b$ se, e somente se, $\alpha a+(1-\alpha) c \unrhd \alpha b+(1-\alpha) c$.

- $\unrhd$ é Afim por Dominância se para todo $a, b, c_{1}, c_{2} \in B_{0}(\Sigma)$ e $\alpha \in(0,1)$, se $a \unrhd b$ e $c_{1} \unrhd c_{2}$, então $\alpha a+(1-\alpha) c_{1} \unrhd \alpha b+(1-\alpha) c_{2}$. Note que essa propriedade diz que $\unrhd$ é um subconjunto convexo de $B_{0}(\Sigma) X B_{0}(\Sigma)$.

- $\unrhd$ é Aditiva quando para todo $a, b, c \in B_{0}(\Sigma)$, se $a \unrhd b$, então $a+c \unrhd b+c$.

\section{A.1.2 Lemas Preliminares}

Vamos agora apresentar as principais demonstrações elaboradas em Faro [8]. Antes de provar o teorema principal da representação PBV, o autor enuncia alguns lemas necessários. 
Lema A.1.1. Uma relação binária $\unrhd$ em $B_{0}(\Sigma)$ Contínua com respeito a misturas e Afim por Dominância é Aditiva.

Demonstração. Considere $a, b, c, d \in B_{0}(\Sigma)$ tais que $a \unrhd b$. Primeiro, assuma que $\lambda(a-b)=c-d$, para algum $\lambda \in(0,1)$. Nesse caso, Afinidade por Dominância implica que

$$
c=\lambda a+(1-\lambda) \frac{1}{1-\lambda}(c-\lambda a) \unrhd \lambda b+(1-\lambda) \frac{1}{1-\lambda}(c-\lambda a)=d
$$

Agora assumimos a igualdade $a-b=c-d$. Note que Continuidade com respeito a misturas implica no ranking $c \unrhd d$ se demonstrarmos a inclusão

$$
(0,1) \subset\{\lambda \in[0,1]: \lambda c+(1-\lambda) d \unrhd d\}
$$

Considere $\lambda \in(0,1)$, já que $\lambda c+(1-\lambda) d-d=\lambda(c-d)=\lambda(a-b)$, o caso anterior nos dá que $\lambda c+(1-\lambda) d \unrhd d$.

Lema A.1.2. Uma relação binária $\unrhd$ em $B_{0}(\Sigma)$ Reflexiva, Afim por dominância, Transitiva sem ambiguidade é Contínua se, e somente se, for Contínua com respeito a misturas.

Demonstração. Claramente, se $\unrhd$ for contínua, então é contínua com respeito a misturas.

Por outro lado, assuma $\unrhd$ contínua com respeito a misturas. Sejam $\left\{a_{n}\right\}_{n \in \mathbb{N}},\left\{b_{n}\right\}_{n \in \mathbb{N}}$ sequências $\|\cdot\|_{\infty^{-}}$convergentes com limites $a, b \in B_{0}(\Sigma)$, respectivamente; tais que para todo índice $n$ tem-se $a_{n} \unrhd b_{n}$.

Note que, pelo lema A.1.1, $\unrhd$ é aditiva. Precisamos mostrar que $a \unrhd b$, o que equivale a mostrar (por aditividade) que $a-b \unrhd 0$, sendo 0 a função constante nula.

Temos para todo $n \geq 1, a_{n} \unrhd b_{n}$. A aditividade de $\unrhd$ nos permite escrever $c_{n}:=a_{n}-b_{n} \unrhd 0$ para todo $n \geq 1$ e $c_{n} \stackrel{\|\cdot\|_{\infty}}{\longrightarrow} a-b=$ : c. Como $1_{S} \leq\left(\|c\|_{\infty}+1\right) 1_{S}-c$, notamos que para todo $\varepsilon \in(0,1)$ existe $m \geq 1$ que satisfaz

$$
c_{m} \leq c+\varepsilon 1_{S} \leq c+\varepsilon\left[\left(\|c\|_{\mathrm{inf}}+1\right) 1_{S}-c\right]
$$

Desse modo,

$$
c+\varepsilon\left[\left(\|c\|_{\infty}+1\right) 1_{S}-c\right]=\varepsilon\left[\left(\|c\|_{\infty}+1\right) 1_{S}\right]+(1-\varepsilon) c
$$

Para todo $\varepsilon \in(0,1)$, existe $m \geq 1$ tal que $c_{m} \leq \varepsilon\left[\left(\|c\|_{\infty}+1\right) 1_{S}\right]+(1-$ $\varepsilon) c$, e como $c_{m} \unrhd 0$, por Transitividade sem Ambiguidade obtemos que, para todo $\varepsilon \in(0,1), \varepsilon\left[\left(\|c\|_{\infty}+1\right) 1_{S}\right]+(1-\varepsilon) c \unrhd 0$.

Sendo assim,

$$
(0,1) \subset\left\{\alpha \in[0,1]: \alpha\left[\left(\|c\|_{\infty}+1\right) 1_{S}\right]+(1-\alpha) c \unrhd 0\right\}=: A
$$

Ainda mais, como $\unrhd$ é contínua com respeito a misturas, a inclusão acima implica que $0 \in A=[0,1]$, i.e., $a \unrhd b$.

Lema A.1.3. Seja $\unrhd$ uma relação binária em $B_{0}(\Sigma)$ Reflexiva, Transitiva sem Ambiguidade e Afim por Dominância. Então $\unrhd$ é Monótona no sentido que, para todo $a, b \in B_{0}(\Sigma)$, se $a(s) \geq(>) b(s), \forall s \in S$; então $a \unrhd(\triangleright) b^{1}$.

\footnotetext{
${ }^{1}$ Note que $a(s) \unrhd b(s) \Leftrightarrow a(s) \geq b(s)$ e $a(s) \triangleright b(s) \Leftrightarrow a(s)>b(s)$
} 
Demonstração. Primeiro, se $\forall s \in S, a(s) \geq b(s)$, então como $\unrhd$ é Reflexiva, $b \unrhd b$; e por Transitividade sem Ambiguidade $a \unrhd b$. Agora suponha que, $\forall s \in S, a(s)>b(s)$. Já sabemos que $a \unrhd b$. Agora suponha que também temos $b \unrhd a$. Escolha $c \in B_{0}(\Sigma)$ tal que $\alpha b+(1-\alpha) c \unrhd \alpha a+(1-\alpha) c$. Agora, seja $a_{*}$ o ato constante que retorna a pior consequência em $\alpha a+(1-\alpha) c$ em todo estado. Isto é, existe $s_{*}$ tal que para todo $s \in S,(\alpha a+(1-$ $\alpha) c)(s) \unrhd a_{*}(s)=(\alpha a+(1-\alpha) c)\left(s_{*}\right)$. Por Transitividade sem Ambiguidade, $\alpha b+(1-\alpha) c \unrhd a_{*}$. Por outro lado, como $\alpha b+(1-\alpha) c=\alpha b\left(s_{*}\right)+(1-\alpha) c\left(s_{*}\right)$ e $\alpha a\left(s_{*}\right)+(1-\alpha) c\left(s_{*}\right)=a_{*}$, por $a\left(s_{*}\right)>b\left(s_{*}\right)$ devemos ter

$$
a_{*}=\alpha a\left(s_{*}\right)+(1-\alpha) c\left(s_{*}\right)>\alpha b\left(s_{*}\right)+(1-\alpha) c\left(s_{*}\right)=\alpha b+(1-\alpha) c
$$

Uma contradição.

Lema A.1.4. Seja $\unrhd$ uma relação binária em $B_{0}(\Sigma)$ Reflexiva, Transitiva sem Ambiguidade, Contínua e Afim por Dominância. Então existe uma preferência de utilidade esperada subjetiva $\unrhd_{q}$ (com respeito a uma probabilidade subjetiva $q \in \Delta$ ) tal que para todo $a, b \in B_{0}(\Sigma)$, se $a \unrhd b$ então $a \unrhd_{q} b$.

Demonstração. Primeiro, vamos definir uma função auxiliar, que será o elemento central para a prova do teorema de representação PBV. Considere um mapa $\eta^{*}: \Delta \rightarrow[0,+\infty]$ definido por $^{2}$, para todo $p \in \Delta$ :

$$
\eta^{*}(p)=\sup _{(a, b) \in \Delta}\left(\int(b-a) d p\right)
$$

Dado o par de funções $(a, b) \in \unrhd$ denotamos o mapa

$$
\begin{gathered}
\tau_{(a, b)}: \Delta \rightarrow \mathbb{R} \\
p \mapsto \tau_{(a, b)}(p):=\int(b-a) d p
\end{gathered}
$$

Note que $\tau_{(a, b)}$ é linear e contínua fraca*. Assim, pelos argumentos usuais $^{3}$, o envelope superior

$$
\eta^{*}=\sup _{(a, b) \in \unrhd} \tau_{(a, b)}(\cdot)
$$

é uma função convexa e fraca* semicontínua inferior. Note também que, como $\unrhd$ é Reflexiva, $\eta^{*} \geq 0$ para todo $p \in \Delta$.

Note que se mostrarmos que existe $q \in \Delta$ tal que $\eta^{*}(q)=0$, então obtemos o resultado desejado pois

$$
\sup _{(a, b) \in \unrhd}\left(\int(b-a) d p\right)=0 \Rightarrow \forall(a, b) \in \unrhd, \int a d p \geq \int b d p
$$

Primeiro, repare que $\inf _{p \in \Delta} \eta^{*}(p)=0$. De fato, se $\unrhd$ é Afim por Dominância e Contínua, $\unrhd$ é um subconjunto convexo e fechado de $B_{0}(\Sigma) X B_{0}(\Sigma)$. Também, pelo Teorema de Banach-Alaoglu-Bourbaki ${ }^{4}, \Delta$ é um subconjunto de $b a(\Sigma)$ fraco* compacto. Ainda mais, é fácil ver que a função $p \longmapsto \tau_{(a, b)}(p)$

\footnotetext{
${ }^{2}$ Considera-se a notação $(a, b) \in \unrhd \Leftrightarrow a \unrhd b$

${ }^{3}$ Veja, por exemplo, Brézis [3], pp. 10-11.

${ }^{4}$ Veja, por exemplo, Brézis [3], p.66
} 
é afim (logo côncava) para cada $p \in \Delta$. Outro fato importante é que, pelo Lema (A.1.3), se $a \unrhd b$, então existe $s_{0} \in S$ tal que $a\left(s_{0}\right) \geq b\left(s_{0}\right)$. Dessa forma, pelo Teorema MiniMax ${ }^{5}$

$\inf _{p \in \Delta} \sup _{(a, b) \in \unrhd} \int(b-a) d p=\sup _{(a, b) \in \unrhd} \inf _{p \in \Delta} \int(b-a) d p=\sup _{(a, b) \in \unrhd} \inf _{s \in S}(b-a)={ }^{((a, a) \in \unrhd)} 0$

já que $\inf _{s \in S}(b-a) \leq 0$.

Finalmente, como $\Delta$ é um subconjunto fraco* compacto de $b a(\Sigma)$ e $\eta^{*}$ é fraca* semicontínua inferior, pelo Teorema de Baire ${ }^{6}$ existe um $q \in \Delta$ com $\eta^{*}(q)=i n f_{p \in \Delta} \eta^{*}=0$. Assim, existe $q \in \Delta$ tal que se $a \unrhd b$, então $a \unrhd_{q} b$, sendo que a relação binária $\unrhd_{q}$ é a preferência de utilidade subjetiva esperada induzida por $q$.

Resta enunciar o Teorema que Faro [8] utiliza na demonstração da unicidade da sua representação principal.

Teorema A.1.5. ${ }^{7}$ Seja $\succsim^{*}$ uma Preferência Bewley Variacional representada pelo par $\left(u, \eta^{*}\right)$ e suponha que $\succsim^{* *}$ é uma pré-ordem completa, monótona e contínua. Se $\succsim^{*} e \succsim^{* *}$ satisfazem ambas Consistência Fraca + Default a Certeza, então

$$
f \succsim^{* *} g \Leftrightarrow \min _{p \in \Delta} \int u(f) d p+\eta^{*}(p) \geq \min _{p \in \Delta} \int u(g) d p+\eta^{*}(p)
$$

\section{A.1.3 Resultado Principal}

Vamos agora a demonstração do teorema 6.3.1:

Demonstração. (1) $\Rightarrow(\mathbf{2})$ :

Primeiro, mostramos que uma representação a la Von Neumann-Morgenstern [18] é válida para as nossas preferências quando restringida a atos constantes. Denote por $\succsim^{c}$ a restrição de $\succsim$ a um conjunto de consequências $X$. Lembre que C-Completude impõe que $\succsim^{c}$ seja completa. Além disso, por Transitividade sem Ambiguidade, temos que $\succsim^{c}$ é transitiva. Também, $\succsim^{c}$ satisfaz Continuidade com respeito a Misturas e Independência Dominada; logo para quaisquer $x, y, z \in X$ e todo $\alpha \in(0,1)$, se $x \succsim^{c} y$ então $\alpha x+(1-\alpha) z \succsim^{c} \alpha y+(1-\alpha) z$. Por Dubra, Maccheroni e Ok [4] as hipóteses do teorema da mistura dos espaços de Hernstein e Milnor [13] são satisfeitas; assim existe uma função afim ${ }^{8} u: X \rightarrow \mathbb{R}$ tal que $x \succsim y$ se, e somente se, $u(x) \geq u(y)$. Além disso, $u$ é única até uma transformação linear positiva. Note que o axioma 6 de não limitação sobre as loterias implica também que $u$ seja sobrejetiva, ou seja, $u(X)=\mathbb{R}^{9}$.

Dado um índice de utilidade $u$, afim e sobrejetivo, podemos definir o mapa:

$$
\Psi_{u}: \mathcal{F} \rightarrow B_{0}(\Sigma)
$$

\footnotetext{
${ }^{5}$ Veja, por exemplo, Aubin and Ekeland [1], capítulo 6.

${ }^{6}$ Veja, por exemplo, Brézis [3], p. 11.

${ }^{7}$ Para demonstração vide Faro[8]

${ }^{8}$ Lembre que $u$ é afim se para todo $x, y \in X$ e $\alpha \in(0,1), u(\alpha x+(1-\alpha) y)=\alpha u(x)+$ $(1-\alpha) u(y)$

${ }^{9}$ Veja, por exemplo, Lema 29 de MMR [16]
} 


$$
f \mapsto \Psi_{u}(f):=u(f)
$$

sendo que $u(f): S \rightarrow \mathbb{R}$ é bem definida por $u(f)(s):=u(f(s))$ para todo $s \in S$. Note que $\Psi_{u}$ é sobrejetiva: se $a \in B_{0}(\Sigma)$, então existe uma partição finita $\left\{E_{k}\right\}_{k=1}^{K}$ e números reais $\left\{r_{k}\right\}_{k=1}^{K}$ tais que $a=\sum_{k=1}^{K} r_{k} 1_{E_{k}}$. Como $u$ é sobrejetiva, existe um conjunto finito de consequências $\left\{x_{k}\right\}_{k=1}^{K}$ tais que $u\left(x_{k}\right)=r_{k}$ para todo $k \in\{1, \ldots, K\}$.

Agora, tome $f$ tal que para todo $k \in\{1, \ldots, K\}, f\left(E_{k}\right)=\left\{x_{k}\right\}$; assim, dado um índice $k, u(f(s))=r_{k}, \forall s \in E_{k}$. Desse modo $\Psi_{u}(f)=a$. Finalmente, note que $u(f)=u(g) \Leftrightarrow u(f(s))=u(g(s)), \forall s \in S \Leftrightarrow f(s) \sim^{c}$ $g(s), \forall s \in S$; e como $\succsim$ é monótona, $f \sim g$. Logo, $\Psi_{u}$ é $\succsim$-injetiva, ou seja, se não $f \sim g$ então $\Psi_{u}(f) \neq \Psi_{u}(g)$. Dado $a \in B_{0}(\Sigma)$, denotamos por $\Psi_{u}^{-1}(a):=\left\{f \in \mathcal{F}: \Psi_{u}(f)=a\right\}$, portanto se $f, g \in \Psi_{u}^{-1}(a)$, então $f \sim g$.

Agora definimos a relação binária $\unrhd$ sobre o conjunto $B_{0}(\Sigma)=\{u(f)$ : $f \in \mathcal{F}\}$ por

$a \unrhd b \Leftrightarrow f \succsim g$, para algum $f, g \in \mathcal{F}$ tal que $a=u(f)$ e $b=u(g)$

Note que $\unrhd$ é bem definida em $B_{0}(\Sigma)$ e

$$
a \unrhd b \Leftrightarrow f \succsim g \text { para todo }(f, g) \in \Psi_{u}^{-1}(a) X \Psi_{u}^{-1}(a)
$$

Por argumentos usuais temos que $\unrhd$ é Reflexiva, Não-Trivial, Transitiva sem Ambiguidade, Contínua com respeito a Misturas e Afim por Dominância. Desse modo, pelos lemas A.1.1 e A.1.2, ฉé Aditiva e Contínua.

Agora, o passo fundamental é usar o mapa $\eta^{*}: \Delta \rightarrow[0,+\infty]$ introduzido no Lema A.1.4 e definido por: para todo $p \in \Delta$

$$
\eta^{*}(p)=\sup _{(f, g) \in \succsim}\left(\int(u(g)-u(f)) d p\right)=\sup _{(a, b) \in \unrhd}\left(\int(b-a) d p\right)
$$

Como $\unrhd$ é Aditiva, notamos que $a \unrhd b$ se, e somente se, $a-b \unrhd 0$, sendo 0 a função constante nula. Assim, colocando $U_{0}:=\left\{c \in B_{0}(\Sigma): c \unrhd 0\right\}$ podemos escrever

$$
\eta^{*}(p)=-\inf _{c \in U_{0}} \int c d p=\sup _{c \in U_{0}} \int-c d p
$$

Assim, como visto no lema A.1.4, $\eta^{*}$ é uma função não negativa, semicontínua inferior fraca* e convexa; tal que $\left\{\eta^{*}=0\right\} \neq \emptyset$.

Agora, vamos mostrar que é possível usar $\eta^{*}$ para representar $\succsim$ conforme dito no teorema.

Se $f_{0} \succsim g_{0}$, então $\eta^{*}(p) \geq \int\left(u\left(g_{0}\right)-u\left(f_{0}\right)\right) d p$ para qualquer $p \in \Delta$. Desse modo,

$$
\int u\left(f_{0}\right) d p+\eta^{*}(p) \geq \int u\left(g_{0}\right) d p, \forall p \in \Delta
$$

Analogamente, se $\left(f_{0}, g_{0}\right) \notin \succsim$, então $\left(a_{0}, b_{0}\right) \notin \unrhd$, sendo $a_{0}=u\left(f_{0}\right)$ e $b_{0}=u\left(g_{0}\right)$. Além disso, definindo $c_{0}:=a_{0}-b_{0}$, obtemos que $c_{0} \notin U_{0}$. Lembre que $U_{0}$ é um subconjunto de $B_{0}(\Sigma)$ não vazio, convexo e fechado; e pelo Teorema da Separação ${ }^{10}$ existe $q \in b a(\Sigma)$ tal que

\footnotetext{
${ }^{10}$ Veja, por exemplo, Dunford e Schwartz [5] , p. 417, ou Brézis [3] , p.7.
} 


$$
\int c_{0} d q<\inf _{c \in U_{0}} \int c d q
$$

Notamos que para todo $E \in \Sigma$, obtém-se $q(E) \geq 0$. De fato, se $q(F)<0$ para algum $F \in \Sigma$, como $n 1_{F} \unrhd 0$ para todo $n \in \mathbb{N}$, vem que

$$
\inf _{c \in U_{0}} \int c d q \leq \inf _{n \in \mathbb{N}} n q(F)=-\infty
$$

uma contradição pois vimos que $\int c_{0} d q \in \mathbb{R}$ é um limitante inferior de $\left\{\int c d q: c \in U_{0}\right\}$. Note que podemos assumir, sem perda de generalidade, $q(S)=1 ;$ ou seja $q \in \Delta$, que implica em

$$
\int c_{0} d q<\inf _{c \in U_{0}} \int c d q=-\eta^{*}(q)
$$

Assim,

$$
\int u\left(f_{0}\right) d q+\eta^{*}(q)<\int u\left(g_{0}\right) d q
$$

que retorna a contrapositiva: se para todo $p \in \Delta$

$$
\int u\left(f_{0}\right) d q+\eta^{*}(q) \geq \int u\left(g_{0}\right) d q
$$

Então $f_{0} \succsim g_{0}$.

$(2) \Rightarrow(1)$ : Demonstração direta.

Unicidade: Dada uma preferência Bewley Variacional $\succsim$ representada por $\left(u, \eta^{*}\right)$, sabemos que $u(X)=\mathbb{R}$. Considere uma preferência variacional $\succsim^{\star}$ também representada pelo par $\left(u, \eta^{*}\right)$. Como $u(X)$ é não limitada sobre as loterias, a Proposição 6 em MMR [16] retorna que o mapa $\eta^{*}$ é a única função em $\mathcal{N}(\Delta)$ tal que

$$
f \succsim^{\star} g \Leftrightarrow \min _{p \in \Delta}\left\{\int u(f) d p+\eta^{*}(p)\right\} \geq \min _{p \in \Delta}\left\{\int u(g) d p+\eta^{*}(p)\right\}
$$

Agora, pelo Teorema (A.1.5) $)^{11}$, é fácil ver que se $\left(u, \eta^{* *}\right)$ também representa $\succsim$, então $\eta^{* *}=\eta^{*}$.

\footnotetext{
${ }^{11}$ Esse resultado não depende de unicidade.
} 


\section{Referências Bibliográficas}

[1] Jean-Pierre Aubin and Ivar Ekeland. Applied nonlinear analysis. 1984. A Wiley-Interscience Publication, New York.

[2] Truman F Bewley. Knightian decision theory. part i. Decisions in economics and finance, 25(2):79-110, 2002.

[3] Haim Brezis. Functional analysis, Sobolev spaces and partial differential equations. Springer Science \& Business Media, 2010.

[4] Juan Dubra, Fabio Maccheroni, and Efe A Ok. Expected utility theory without the completeness axiom. Journal of Economic Theory, 115(1):118-133, 2004.

[5] N. Dunford and J.T. Schwartz. Linear Operators, Part I: General Theory. Wiley, New York, 1958.

[6] Daniel Ellsberg. Risk, ambiguity, and the savage axioms. The quarterly journal of economics, pages 643-669, 1961.

[7] Daniel Ellsberg. Risk, ambiguity, and decision. Taylor \& Francis, 2001.

[8] José Heleno Faro. Variational bewley preferences. Journal of Economic Theory, 157:699-729, 2015.

[9] Paolo Ghirardato, Fabio Maccheroni, and Massimo Marinacci. Differentiating ambiguity and ambiguity attitude. Journal of Economic Theory, 118(2):133-173, 2004.

[10] Itzhak Gilboa, Fabio Maccheroni, Massimo Marinacci, and David Schmeidler. Objective and subjective rationality in a multiple prior model. Econometrica, 78(2):755-770, 2010.

[11] Itzhak Gilboa and Massimo Marinacci. Ambiguity and the bayesian paradigm. Chapter, 7:179-242, 2011.

[12] Itzhak Gilboa and David Schmeidler. Maxmin expected utility with non-unique prior. Journal of mathematical economics, 18(2):141-153, 1989.

[13] Israel N Herstein and John Milnor. An axiomatic approach to measurable utility. Econometrica, Journal of the Econometric Society, pages 291-297, 1953.

[14] Anscombe F. J. and R. Aumann. A definition of subjective probability. Annals of Mathematical Statistics, 34:199-205, 1963.

[15] Frank H. Knight. Risks, uncertainty and profit, 1921. 
[16] Fabio Maccheroni, Massimo Marinacci, and Aldo Rustichini. Ambiguity aversion, robustness, and the variational representation of preferences. Econometrica, pages 1447-1498, 2006.

[17] Leonard J Savage. The foundations of statistics. Courier Corporation, 1972.

[18] John Von Neumann and Oskar Morgenstern. Theory of games and economic behavior. 1944 . 